\title{
Evidence for Deleterious Antigenic Imprinting in SARS-CoV-2 Immune Response
}

\author{
Sanjana R. Sen ${ }^{1}$, Emily C. Sanders ${ }^{2}$, Alicia M. Santos ${ }^{2}$, Keertna Bhuvan², Derek Y. Tang ${ }^{2}$, \\ Aidan A. Gelston², Brian M. Miller ${ }^{2}$, Joni L. Ricks-Oddie ${ }^{3,4}$ and Gregory A. Weiss ${ }^{1,2,5 *}$
}

\section{Author Affiliations:}

1 Department of Molecular Biology \& Biochemistry, University of California, Irvine, Irvine CA 92697-3900 USA

2 Department of Chemistry, University of California, Irvine, Irvine CA 92697-2025 USA

${ }^{3}$ Center for Statistical Consulting, Department of Statistics, University of California, Irvine, Irvine CA 92697-1250 USA

4 Biostatics, Epidemiology and Research Design Unit, Institute for Clinical and Translational Sciences, University of California, Irvine, Irvine CA 92697-4094 USA

5 Department of Pharmaceutical Sciences, University of California, Irvine, Irvine CA 92697-3958 USA

*Address correspondence to gweiss@uci.edu

Corresponding Author:

*GAW e-mail: gweiss@uci.edu, Tel: +1-949-824-5566, address: 1102 Natural Sciences 2, Irvine, CA 92697-2025, USA

\section{Author Contributions:}

S.R.S., and G.A.W. designed research; S.R.S., E.C.S., A.M.S., K.B., D.Y.T, A.A.G., and B.M.M. performed research; S.R.S., E.C.S. and G.A.W. analyzed data; J.L.R. advised on statistical analysis; S.R.S. and G.A.W. wrote the manuscript.

\section{Competing Interests:}

None.

\section{Acknowledgements:}

We thank Professor Elizabeth Bess and Professor Stacey Schultz-Cherry for helpful conversations, Kristin Gabriel for initial bioinformatics analysis, and the patients who generously donated their plasma samples (IRB\# 2012-8716). We gratefully acknowledge the support of the UCI COVID-19 Basic, Translational and Clinical Research Fund (CRAFT), the Allergan Foundation, and UCOP Emergency COVID-19 Research Seed Funding. A.M.S thank the Minority Access to Research Careers (MARC) Program, funded by the NIH (GM-69337). J.L.R.-O. was supported by the National Center for Research Resources and the National Center for Advancing Translational Sciences from the NIH (TR001414). 


\section{Abstract}

A previous report demonstrated the strong association between the presence of

41 antibodies binding to an epitope region from SARS-CoV-2 nucleocapsid, termed Ep9, and

42 COVID-19 disease severity. Patients with anti-Ep9 antibodies (Abs) had hallmarks of antigenic

43 imprinting (AIM), including early $\lg G$ upregulation and cytokine-associated injury. Thus, the

44 immunological memory of a previous infection was hypothesized to drive formation of suboptimal

45 anti-Ep9 Abs in severe COVID-19 infections. This study identifies a putative primary antigen

46 capable of stimulating production of cross-reactive, anti-Ep9 Abs. Binding assays with patient

47 blood samples directly show cross-reactivity between Abs binding to Ep9 and only one

48 bioinformatics-derived, homologous potential antigen, a sequence derived from the

49 neuraminidase protein of H3N2 Influenza A virus. This cross-reactive binding is highly influenza

50 strain specific and sensitive to even single amino acid changes in epitope sequence. The

51 neuraminidase protein is not present in the influenza vaccine, and the anti-Ep9 Abs likely resulted

52 from the widespread influenza infection in 2014. Therefore, AIM from a previous infection could

53 underlie some cases of COVID-19 disease severity.

\section{Importance}

55 Infections with SARS-COV-2 result in diverse disease outcomes, ranging from asymptomatic to

56 fatal. The mechanisms underlying different disease outcomes remain largely unexplained.

57 Previously, our laboratory identified a strong association between the presence of an antibody

58 and increased disease severity in a subset of COVID-19 patients. Here, we report that this

59 severity-associated antibody cross-reacts with viral proteins from an influenza A viral strain from

60 2014. Therefore, we speculate that antibodies generated against previous infections, like the 2014

61 influenza A, play a significant role in directing some peoples' immune responses against SARS-

62 COV-2. Such understanding of the sources and drivers of COVID-19 disease severity can help 63 early identification and pre-emptive treatment. 


\section{4}

65

66

67

68

69

70

71

72

73

74

75

$87 \quad \alpha \mathrm{Ep} 9(+)$ patients.

\section{Introduction}

Original antigenic sin or antigenic imprinting (AIM) occurs when the immune response adapted for a primary (or "original”) infection instead targets a similar, but not identical, pathogen ${ }^{1}$. Since B-cells undergo affinity maturation post-primary infection, cross-reactive Abs from previous infections can outcompete naïve $\mathrm{Abs}^{2}$. AIM ideally accelerates pathogen clearance by targeting highly conserved antigens; however, suboptimal targeting by non-neutralizing, Ab binding can exacerbate disease ${ }^{2}$. The range of outcomes observed in COVID-19, from asymptomatic to fatal, could result from a patient's immunological memory ${ }^{1,3}$.

Ab cross-reactivity from AIM causes a wide range of disease outcomes. For example, some Abs from healthy individuals previously exposed to other common human coronaviruses (hCoV) could cross-react with SARS-CoV-2 spike protein to neutralize viral pseudotypes ${ }^{4}$. However, other prepandemic Abs with cross-reactivity to SARS-CoV-2 nucleocapsid (NP) and spike proteins did not protect against severe symptoms ${ }^{5}$. Humoral immunity to hCoVs, NL63 and $229 \mathrm{E}^{6}$, respiratory syncytial virus, cytomegalovirus and herpes simplex virus $-1^{7,8}$ has been associated with more severe COVID-19 disease.

The presence of Abs with affinity for a 21-mer peptide derived from SARS-CoV-2 NP, an epitope region termed Ep9, have been correlated with severe COVID-19. The patients, termed $\alpha$ Ep9 $(+)$, comprised $\approx 27 \%$ of the sampled, SARS-CoV-2-infected population $(n=186)$. The aEp9(+) patients $(n=34)$ had high, early levels of $\alpha \mathrm{N}$ IgGs, typically within the first week, compared to aEp9(-) patients; $\alpha \mathrm{Ep} 9(+)$ individuals also experienced cytokine-related, immune hyperactivity ${ }^{9}$. These two observations suggest an AIM-based mechanism for the disease severity observed in aEp9(+) patients. Here, we explore the epitope homology landscape and aEp9 Ab cross-reactivity to potentially identify an original antigen driving Ab-based immune response in 


\section{Results and Discussion}

Assays measured levels of $\alpha$ Ep9 lgGs and IgMs from aEp9(+) patients whose plasma was collected at various times post-symptom onset (PSO). Consistent with the hallmarks of AIM tracing a prior infection, aEp9 IgG levels appeared elevated as early as one day PSO in one patients. Similar IgG levels were observed in the patient population over $>4$ weeks (one-way ANOVA, $p=0.321$ ); thus, $\alpha E p 9$ IgG started high and remained high. Levels of $\alpha$ Ep9 IgMs amongst patients at various times PSO were also similar (one-way ANOVA, $p=0.613$ ). The signals measured for $\alpha \mathrm{Ep} 9$ IgM levels were significantly lower than the equivalent $\alpha \mathrm{Ep} 9 \lg$ levels (t-test, $\mathrm{p}=0.0181$ ) (Figure $\mathrm{S} 1 \mathrm{~A})$; this difference could reflect lower IgM affinity, quantity, or both.

Searches for sequence and structural homologs of Ep9 using $\mathrm{pBLAST}^{10}$ and $\mathrm{VAST}^{11}$ databases suggested candidate primary antigens. A structural homolog from betaherpesvirus $6 \mathrm{~A}$ and 14 other Ep9 sequence homologs were identified. Additionally, Ep9-orthologous regions from six human coronaviruses (SARS-CoV, MERS, OC43, HKU-1, NL63, 229E) were chosen for subsequent assays (Figure 1A, and Table S1). To expedite the binding measurements, the potential AIM epitope regions were subcloned into phagemids encoding the sequences as fusions to the M13 bacteriophage P8 coat protein. DNA sequencing and ELISA experiments demonstrated successful cloning and consistent phage display, respectively. Two epitopes failed to display on phage and were omitted from subsequent investigation (Table S2 and Figure S2A).

Phage ELISAs tested binding by Ep9 homologs to aEp9 Abs. An average response within the patient population was assessed using pooled plasma from three sets of five $\alpha \mathrm{Ep} 9(+)$ and five aEp9(-) COVID-19 patients coated onto ELISA plates. Plasma from healthy individuals provided an additional negative control. Confirming previously reported results, SARS-COV-2 Ep9 and a

111 homologous epitope from SARS-CoV-1 (90\% similarity) bound only to plasma from aEp9(+) 112 patients ${ }^{9}$. The aEp9 Ab affinity for SARS-CoV-1 is unlikely to drive SARS-CoV-2 AIM due to the 113 former's limited spread in the US ${ }^{12}$. 
The panel of potential epitopes revealed a candidate epitope from the neuraminidase (NA) protein of an H3N2 influenza A strain, which circulated in 2014 (A/Para/128982-IEC/2014, Accession No. AIX95025.1), termed EpNeu here. The plasma from aEp9(+), but not aEp9(-) 117 patients nor healthy individuals, bound EpNeu ( $p<0.0001$, two-way ANOVA ad hoc Tukey test) 118 (Figure 1C, D). Though Ep9 and EpNeu share 38\% amino acid sequence similarity, other 119 candidate epitope regions with significantly higher homology failed to bind to aEp9(+) plasma 120 (Table S1).

Next, the specificity of aEp9 Abs binding to NA from different viral strains was explored.

122 EpNeu provided a template for further homolog searches in sequence databases. Closely aligned NA sequences isolated from human, avian, and swine hosts in North America were chosen for further analysis (Figure 1F, Table S1). The sequences were phage-displayed as before. Despite their close similarity to EpNeu (up to $92.3 \%$ similarity or only one residue difference), none of the EpNeu homologs bound to Abs from aEp9(+) patients (Figure 1E). A single EpNeu amino acid substitution, K142N (numbering from full-length NA, Accession No. AID57909.1) in an H1N2 swine flu (2016) dramatically decreased binding affinity to Abs from aEp9(+) patients $(p<0.0001$ one-way ANOVA ad hoc Tukey). An epitope H4N6 avian influenza A (2010) missing residue S141, but including conserved K142, also greatly reduced binding to Abs from aEp9(+) patients $(p<0.0001$ one-way ANOVA ad hoc Tukey) (Figure 1E, 1F). Therefore, S141 and K142 are critical 132 for binding to aEp9 Abs.

Do Ep9 and EpNeu epitopes bind the same Abs? Data from 34 aEp9(+) patients demonstrated a strong, highly significant correlation between levels of Abs binding to Ep9 and EpNeu epitopes in patient plasma (Figure 2A). Cross-reactivity was confirmed by a sandwichformat assay requiring bivalent, simultaneous binding to both eGFP-fused Ep9 and phage137 displayed EpNeu (Figure 2B, S3). Cross-reactive Ab binding both Ep9 and EpNeu epitopes in 
pooled plasma from aEp9(+) patients, but not in aEp9(-) patients with other aNP Abs or healthy donors was demonstrated. Thus, we conclude that aEp9 Abs also recognize the EpNeu epitope.

We then investigated whether EpNeu could present a viable antigen during infection with 2014 H3N2 (NCBI: txid1566483). Linear epitope analysis of full-length NA protein (Bepipred 2.0) ${ }^{13}$ predicted a candidate antigen with eight residues from EpNeu, including S141 and K142, and ten additional residues (146-155). This predicted epitope region, termed EpPred, includes the conserved catalytic NA residue D151 targeted for viral neutralization by the immune system ${ }^{14}$ (Figures 2C, and S4A). A model structure of 2014 H3N2 NA from Swiss-Model ${ }^{15}$ and structural epitope prediction (Discotope 2.0) ${ }^{16}$ also identified potential epitopes within EpPred (Figures 2C, D and S4B).

eGFP-fused EpPred (Figure S2B) was assayed with pooled plasma from five aEp9(+) patients. Controls included EpNeu and Ep9 (positive) and eGFP FLAG (negative). The aEp9 Abs bound to Ep9 with $\approx 2$-fold stronger apparent affinity than for EpNeu (Figure 2E). The increased binding strength of Ep9 could result from additional rounds of Ab affinity maturation after the primary infection². The longer length EpPred modestly improved upon the binding of EpNeu to $\alpha$ Ep9 Abs (Figure 3C). Thus, aEp9 Abs likely target a larger epitope of H3N2 2014 NA beyond regions homologous to Ep9; full-length NA's balkiness to overexpression makes this difficult to test ${ }^{17}$. Additionally, the bacterially overexpressed epitopes assayed here do not include post-translational modifications. Taken together, the results support the hypothesis that $\alpha$ Ep9 Abs found in severe COVID-19 disease can result from AIM with H3N2 influenza A. Unfortunately, patient histories typically do not include influenza infections and vaccinations. Isolated from Para, Brazil, the H3N2 2014 strain has unknown spread in North America. However, a severe outbreak of influenza A was recorded in $2014^{18,19}$. Since only hemagglutinin was sequenced for strain identification in $2014^{19}$, the candidate AIM strain from the current investigation could not be effectively traced as only its NA sequence was available. Notably, the EpNeu homolog from the 
164 not bind aEp9 Abs (Figure 1 E, F). Therefore, $\alpha$ EpNeu Abs must have been generated against a 165 primary influenza infection, not the vaccine.

167 COVID-19 in aEp9(+) patients. Specifically, we demonstrate cross-reactive binding between

168 aEp9 Abs and a predicted NA epitope from a 2014 influenza A strain. Future studies could 169 examine correlation between a country's rate of the H3N2 2014 influenza and severe COVID-19.

170 Additionally, correlation could be tested using health systems that record influenza infections.

171 Examining epitope conservation and Ab cross-reactivity could predict AIM-based immune 172 responses and disease outcomes in future infections. Identifying detrimental, benign or beneficial 173 AIM pathways could also guide vaccine design. 


\section{Figures (main text)}
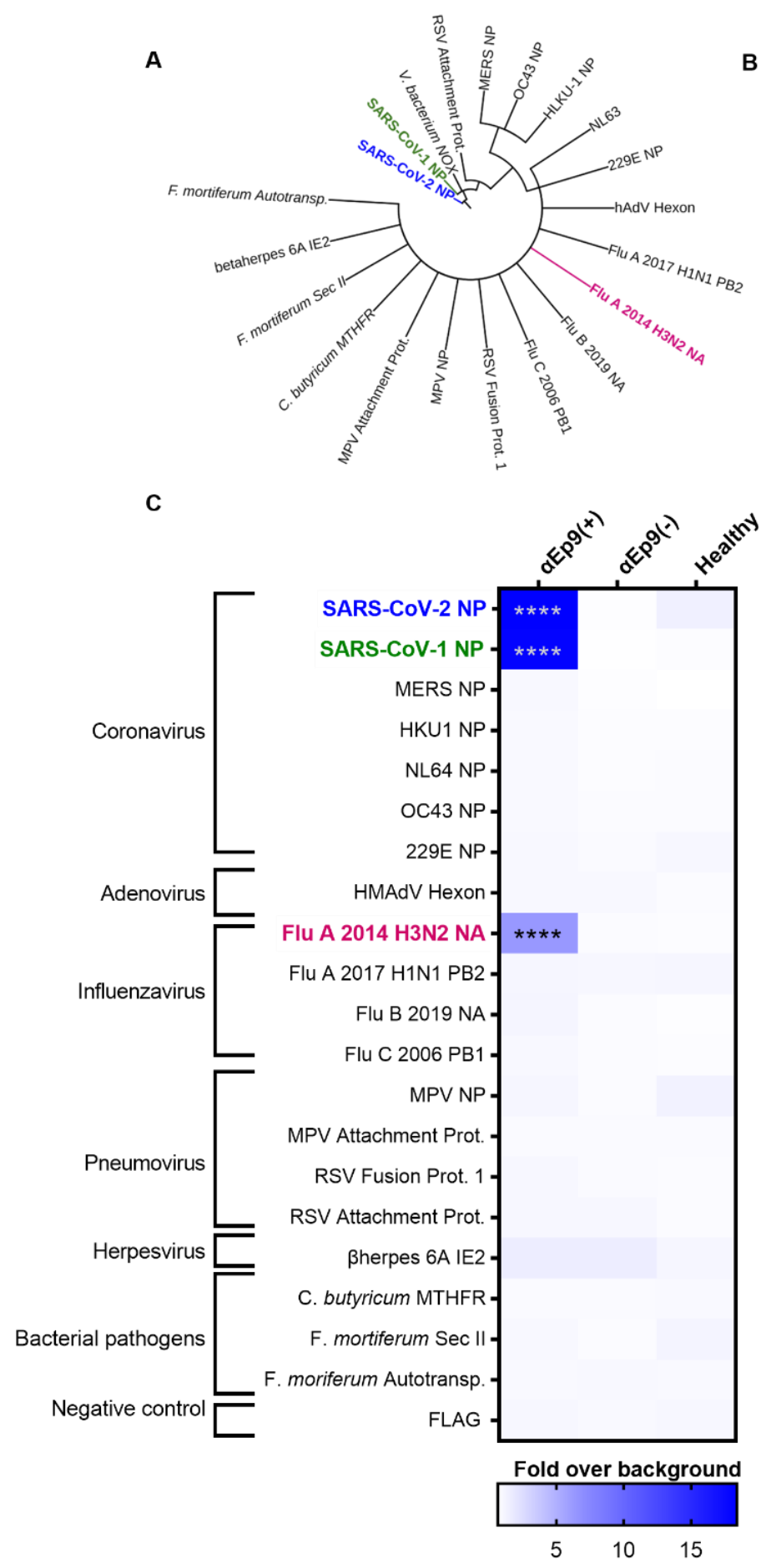

B

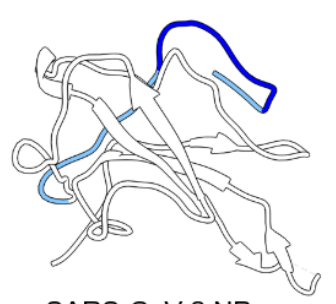

$\mathrm{N}$-terminus domain PDB: 6M3M
SARS-CoV-2 NP,

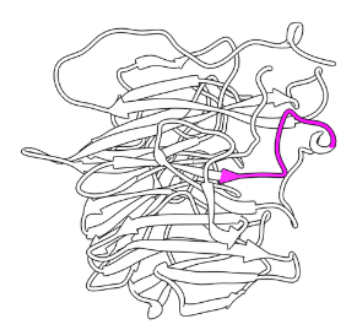

Flu A 2014 H3N2 NA Swiss model PDB

D

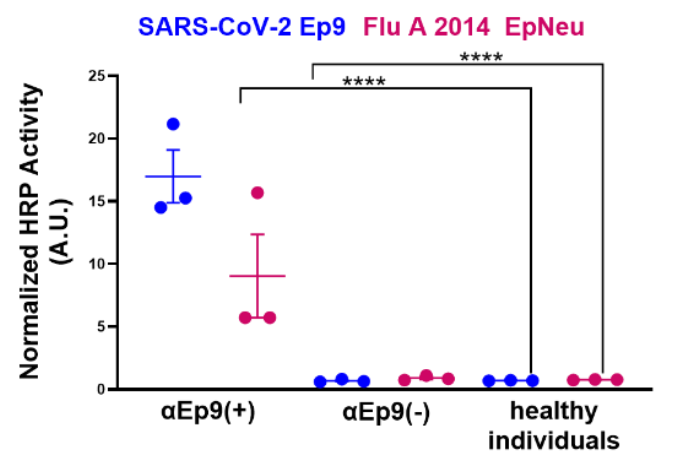

E

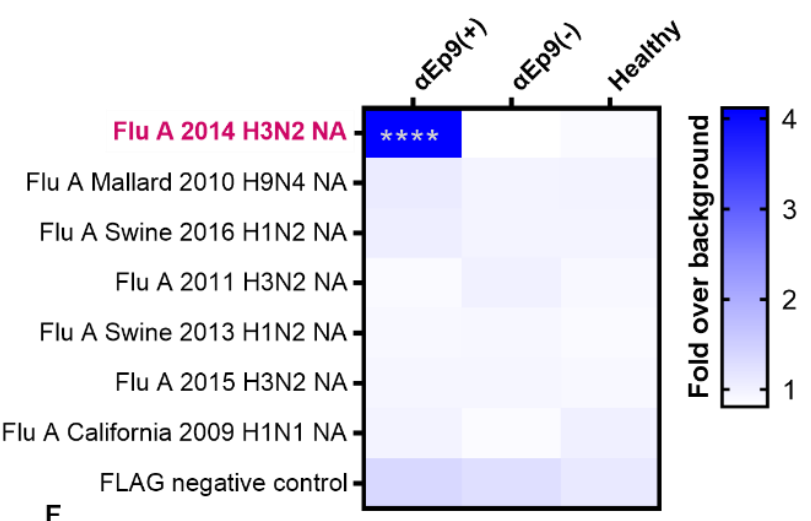

$\mathbf{F}$

\begin{tabular}{|l|l|}
\hline Epitope Source & Sequence \\
\hline SARS-CoV-2 NP (Ep9) & -LPQGTTLLRG-- \\
\hline Flu A 2014 H3N2 NA (EpNeu) & ALGQGTTLSKGHS \\
\hline Flu A Mallard 2010 H9N4 NA & ALSQGTTL_KGHS \\
\hline Flu A Swine 2016 H1N2 NA & ALGQGTTLSNGHS \\
\hline Flu A 2011 H3N2 NA & ALGQGTTLSNVHS \\
\hline Flu A Swine 2013 H1N2 NA & ALGQGTTLLNNGHS \\
\hline Flu A 2015 H3N2 NA & ALGQGTTLNNVHS \\
\hline Flu A 2009 H1N1 NA & FLTQGALLLNDKHS \\
\hline
\end{tabular}


SARS-CoV-2 to the bioinformatics-identified, closest homologs. Sequence alignments used pBLAST and VAST, and the cladogram was generated by iTOL ${ }^{20}$. (B) Structures of SARS-CoV2 NP RNA binding domain (PDB: 6M3M) and the Flu A 2014 H3N2 NA protein (modeled by SWISS-Model ${ }^{21}$ ). SARS-CoV-2 NP highlights Ep9 residues (light and dark blue) and the region homologous region to EpNeu (dark blue). The depicted model of Flu A 2014 H3N2 NA highlights the EpNeu putative antigen (pink). (C) ELISAs examined binding of phage-displayed potential OAS epitopes to Abs from three sets of pooled plasma from five aEp9(+) patients, or five aEp9() patients. Pooled plasma from healthy patients was an additional negative control. The colors of the heat map represent the mean binding signal normalized to phage background negative controls (signal from phage without a displayed peptide). (D) Expansion of data from panel C shows ELISA signals from the independently assayed individual pools shows results from the individual pools ( ${ }^{* * *} \mathrm{p}<0.0001$ for a two-way ANOVA comparing binding of phage-displayed epitopes listed in panel $C$ to different groups of pooled plasma, ad hoc Tukey test). (E) Using EpNeu as the search template to generate homologous sequences (shown in next panel), ELISAs examined EpNeu homologs' binding to pooled plasma from aEp9(+), aEp9(-), or healthy individuals. The data are represented as described in panel $C\left({ }^{* * * *} p<0.0001\right.$ for two-way ANOVA c phage-displayed epitopes, ad hoc Tukey and Dunnett's test as shown). (F) Amino acid sequence alignment of the closely related Flu A NA homologs of EpNeu from pBLAST. Blue and orange residues represent conserved and mismatched amino acids, respectively, relative to Ep9. Bolded residues are important for epitope recognition by aEp9 Abs. Here, the term Flu refers to influenza. 
A B

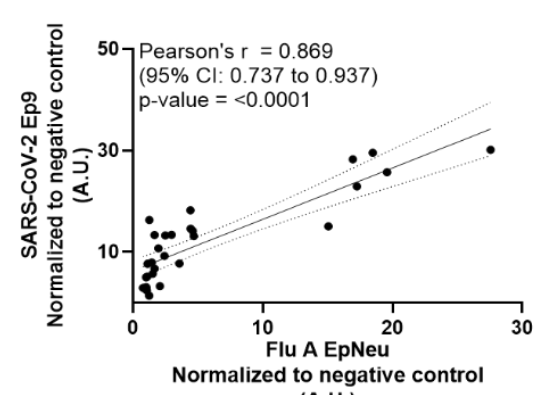

(A.U.)

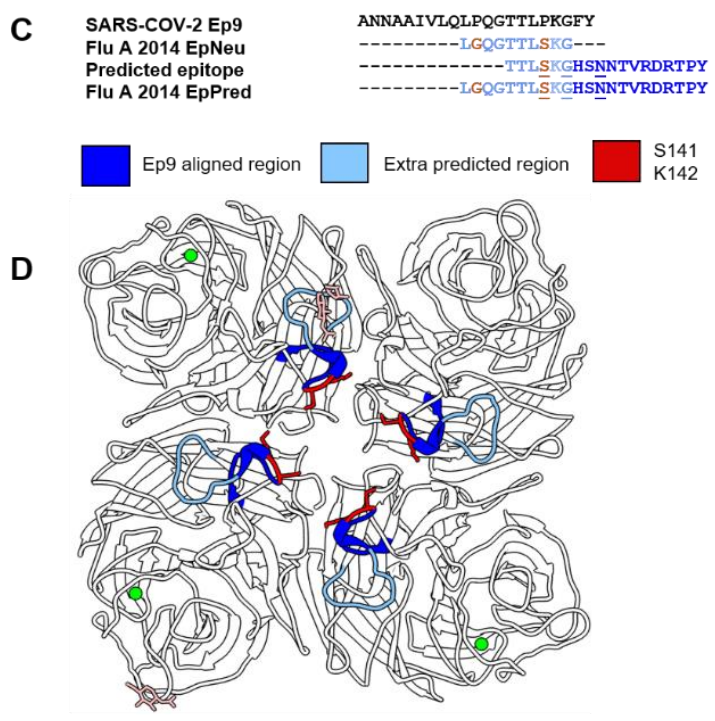

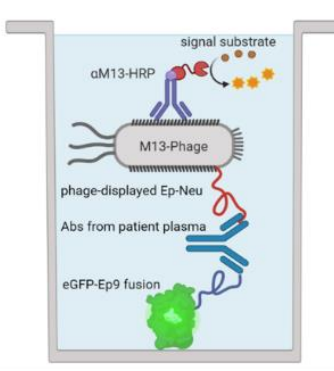

E

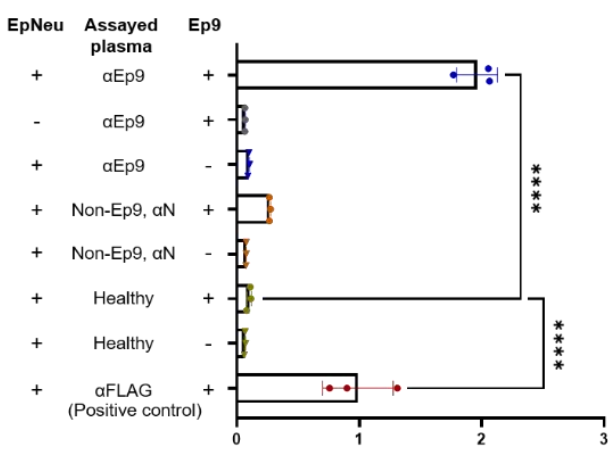

ELISA HRP activity levels (A.U.)

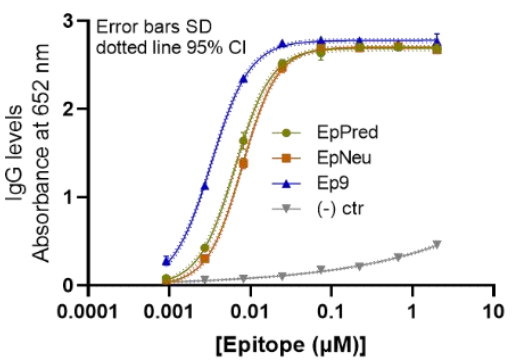

\begin{tabular}{|c|c|c|c|}
\hline Parameter & Flu A EpPred & Flu A EpNeu & SARS-CoV-2 Ep9 \\
\hline $\mathrm{B}_{\max }$ & $2.69(2.66-2.72)$ & $2.71(2.68-2.73)$ & $2.78(2.76-2.80)$ \\
\hline Hill coeff. & $1.93(1.79-2.08)$ & $2.01(1.89-2.13)$ & $1.83(1.73-1.95)$ \\
\hline $\mathrm{EC}_{50}(\mathrm{nM})$ & $6.51(6.23-6.80)$ & $7.94(7.70-8.19)$ & $3.30(3.18-3.43)$ \\
\hline $\mathrm{R}^{2}$ & 0.998 & 0.999 & 0.998 \\
\hline
\end{tabular}

223

224

225

226

227

228

229

230

231

232

233

234

235

236

237

238

239

240

Figure 2 | Cross-reactive Ab binding to both Ep9 and EpNeu, and EpNeu epitope prediction. (A) Comparing normalized levels of phage-displayed Ep9 and EpNeu binding to plasma-coated wells from individual $\alpha \operatorname{Ep} 9(+)$ patients $(n=34)$. A strong correlation is observed, as shown by the depicted statistics. Each point in panels $A$ through $C$ represents data from individual patients. (B) A schematic diagram of the sandwich ELISA to examine cross-reactivity of aEp9 Abs. The assay tests for bivalent Ab binding to both Ep9 and EpNeu. Pooled plasma from five aEp9(+) patients or five $\alpha \mathrm{Ep} 9(-)$ patients with other aNP Abs was tested for bivalent binding to both eGFP-fused Ep9 and phage-displayed EpNeu. Healthy patient plasma was used as a negative control. For additional negative controls, phage-FLAG and eGFP-FLAG replaced Ep9 and EpNeu, respectively $\left({ }^{* * *} p<0.0001\right.$ one-way ANOVA, ad hoc Tukey and Dunnett's test shown, with healthy plasma in the presence of EpNeu and Ep9 as negative control). Error bars represent SD. Individual points on bar graph represent technical replicates. (C) Linear and structural B-cell epitope prediction tools Bepipred 2.0 and Discotope 2.0 suggested an extended, linear epitope region from the influenza A H3N2 $2014 \mathrm{NA}$, including the eight residues of Ep9 Neu (light blue) with an additional ten, C-terminal residues (dark blue). This extended, predicted epitope is termed EpPred. Structural epitope predictions are underlined. Residues on EpNeu that are not aligned with Ep9 are depicted in orange. (D) Structural model depicting the influenza A H3N2 2014 NA. 
241 The model was generated using SWISS-Model based on the NA structure from influenza A H3N2

242 Tanzania 2010 (PDB: 4GZS). The NA structure highlights the EpNeu region (light blue), the

243 extended residues in EpPred (dark blue), potential glycosylation sites (light pink), and the residues

244 S141 and K142 (red), which are important for aEp9 Ab recognition. (E) Dose-dependent ELISA

245 comparing binding of aEp9 Abs to Ep9, EpNeu and EpPred. Pooled plasma from five aEp9(+)

246 patients and five aEp9(-) patients were tested in triplicates with varying concentrations of eGFP-

247 fused epitopes. The data demonstrates the strongest interactions occurred between aEp9 Abs

248 and Ep9 with an approximately 2-fold decrease in aEp9 Abs binding affinity for EpNeu. EpPred

249 bound slightly stronger to aEp9 Abs than EpNeu; the difference in trend lines of EpNeu and

250 EpPred are statistically significant ( $p<0.0001$, Comparison of Fits). Trendlines represent non-

251 linear regression fit with Hill slope analysis. 


\section{Supplementary Materials}

254

255

256

257

258

259

260

261

262

263

264

265

\section{Materials and Methods \\ Sequence and structural alignment analysis}

To identify possible sources of primary infection responsible for $\alpha$ Ep9 Ab generation, sequence and structural alignment with Ep9 residues and the SARS-CoV-2 NP was conducted. Alignment of Ep9 sequence with the orthologs from other human coronaviruses (hCoVs) such as SARSCoV, MERS, HKU-1, NL63, 229E and OC43 was conducted using the Benchling sequence alignment tool ${ }^{22}$ (https://benchling.com). To explore a wider range of human host pathogens pBLAST ${ }^{10}$ (https://blast.ncbi.nlm.nih.gov/Blast.cgi) was used to search for Ep9 homology in a database of non-redundant protein sequences; common human-host viruses were specified in the organism category. The queries were conducted with the blastp (protein-protein BLAST) program ${ }^{10}$ with search parameters automatically adjusted for short input sequences. Alignments spanning $>7$ residues were included here. The Vector Alignment Search Tool (VAST) ${ }^{11}$ (https://structure.ncbi.nlm.nih.gov/Structure/VAST/vast.shtml) was used to find structural alignment between SARS-CoV-2 Ep9 and proteins from other viral and bacterial human host pathogens. Alignment for NP from common hCoV were not further examined, as they had been included in sequence alignment analysis. The aligned sequences were sorted by the number of aligned residues as well as root-mean square deviation (RMDS). The top 50 structurally aligned proteins were then examined for structural homology in the Ep9 epitope region. Regions of proteins that aligned with the Ep9 region were selected for subsequent analysis.

\section{Cloning}

Predicted AIM epitopes were subcloned for phage display using the pM1165a phagemid vector ${ }^{23}$ with an N-terminal FLAG-tag and a C-terminal P8 M13-bacteriophage coat protein. AIM constructs were subcloned using the Q5 site-directed mutagenesis kit (New England Biolabs, 
Ipswich, MA) as per manufacturer's instructions. After cloning, cells were transformed into XL-1

Blue E. coli and spread on carbenicillin-supplemented $(50 \mu \mathrm{g} / \mathrm{ml})$ plates. Individual colonies were then inoculated into $5 \mathrm{ml}$ cultures, and shaken overnight at $37^{\circ} \mathrm{C}$. The phagemid was isolated using the QIAprep spin miniprep kit (Qiagen, Germantown, MD) as per manufacturer's instructions. Cloned sequences were verified by Sanger sequencing (Genewiz, San Diego, CA).

\section{Phage propagation and purification}

The Ep9 homologs were expressed as N-terminal fusions to the P8 coat protein of M13 bacteriophage. Plasmids were transformed into SS320 E. coli and spread onto carbenicillinsupplemented $(50 \mu \mathrm{g} / \mathrm{ml})$ LB-agar plates before overnight incubation at $37^{\circ} \mathrm{C}$. A single colony was inoculated into a primary culture of $15 \mathrm{ml}$ of $2 Y T$ supplemented with $50 \mu \mathrm{g} / \mathrm{ml}$ carbenicillin and $2.5 \mu \mathrm{g} / \mathrm{ml}$ of tetracycline, and incubated at $37^{\circ} \mathrm{C}$ with shaking at $225 \mathrm{rpm}$ until an optical density at $600 \mathrm{~nm}\left(\mathrm{OD}_{600}\right)$ of 0.5 to 0.7 was reached. $30 \mu \mathrm{M}$ IPTG and M13KO7 helper phage at ${ }^{\circ} \mathrm{C}$ with shaking at $225 \mathrm{rpm}$ for $45 \mathrm{~min} .8 \mathrm{ml}$ of the primary culture was then transferred to $300 \mathrm{ml}$ of 2 YT supplemented with $50 \mu \mathrm{g} / \mathrm{ml}$ of carbenicillin and $20 \mu \mathrm{g} / \mathrm{ml}$ of kanamycin. The cultures were inoculated at $30{ }^{\circ} \mathrm{C}$ with shaking at $225 \mathrm{rpm}$ for around $19 \mathrm{~h}$.

The phage propagation culture was centrifuged at $9632 \times \mathrm{g}$ for $10 \mathrm{~min}$ at $4{ }^{\circ} \mathrm{C}$. The supernatant, containing the phage, was transferred into a separate tubes pre-aliquoted with $1 / 5^{\text {th }}$ volume of phage precipitation buffer (20\% w/v PEG-8000 and $2.5 \mathrm{M} \mathrm{NaCl})$, and incubated on ice

299 for $30 \mathrm{~min}$. The solution, containing precipitated phage, was centrifuged for $15 \min$ at $4{ }^{\circ} \mathrm{C}$, and 300 the supernatant was discarded. The precipitated phage was centrifuged a second time at 1,541

$301 \times \mathrm{g}$ for $4 \mathrm{~min}$ at $4{ }^{\circ} \mathrm{C}$, and then dissolved in $20 \mathrm{ml}$ of resuspension buffer (10 $\mathrm{mM}$ phosphate, 137 $302 \mathrm{mM} \mathrm{NaCl}, \mathrm{pH} 7.4-8.0$ with Tween-20 0.05\%, v/v and glycerol 10\% v/v). The resuspended pellet 303 solution was divided into $1 \mathrm{ml}$ aliquots, which were flash frozen with liquid nitrogen for storage in $304-80^{\circ} \mathrm{C}$. Prior to use in ELISA binding assays, the aliquoted phage-displayed constructs were re- 
precipitated in $0.2 \mathrm{ml}$ of phage precipitation buffer after incubation for $30 \mathrm{~min}$ on ice. Aliquots were centrifuged at $12298 \times \mathrm{g}$ for $20 \mathrm{~min}$ at $4^{\circ} \mathrm{C}$ and the supernatant was discarded. The phage pellets were re-centrifuged at $1968 \times \mathrm{g}$ for $4 \mathrm{~min}$ at $4{ }^{\circ} \mathrm{C}$, and then resuspended in $1 \mathrm{ml}$ of $10 \mathrm{mM}$ phosphate, $137 \mathrm{mM} \mathrm{NaCl}, \mathrm{pH} 7.4$.

\section{Expression and Purification of eGFP fusion peptides}

pET28c plasmids encoding eGFP fusions to C-terminal Ep9-FLAG, EpNeu-FLAG, EpPred-FLAG and FLAG (negative control) and N-terminal His 6 peptide epitopes, were transformed into BL21DE3 Star E. coli chemically competent cells. Transformants were spread on carbenicillin-supplemented (50 $\mathrm{g} / \mathrm{ml}) \mathrm{LB}$-agar plates and incubated at $37^{\circ} \mathrm{C}$ overnight. Single colonies of each construct were selected to inoculate $25 \mathrm{ml}$ LB media supplemented with carbenicillin $(50 \mu \mathrm{g} / \mathrm{ml})$. After incubation at $37^{\circ} \mathrm{C}$ with shaking at $255 \mathrm{rpm}$ overnight, $5 \mathrm{ml}$ of seed cultures were used to inoculate $500 \mathrm{ml}$ of LB media supplemented with carbenicillin $(50 \mu \mathrm{g} / \mathrm{ml})$. Expression cultures were incubated at $37{ }^{\circ} \mathrm{C}$ with shaking at $225 \mathrm{rpm}$ until an $\mathrm{OD}_{600}$ of $\sim 0.5$ was reached. The cultures were induced with $0.5 \mathrm{mM} \mathrm{IPTG}$ and incubated at $25^{\circ} \mathrm{C}$ for $18 \mathrm{~h}$. The cells were pelleted by centrifugation at $9632 \times \mathrm{g}$ for $20 \mathrm{~min}$ and resuspended in Tris-HCl lysis buffer (20 mM Tris- $\mathrm{HCl}, 250 \mathrm{mM} \mathrm{NaCl}, \mathrm{pH}$ 8). Cells were lysed by sonication and the insoluble fractions were pelleted by centrifugation at $24696 \times g$. The supernatant was affinity-purified using Profinity ${ }^{\mathrm{TM}}$ IMAC (BioRad, Hercules, CA) resin charged with nickel sulfate. The protein lysate was batch bound overnight to the IMAC resin and purified using gravity columns. Columns were washed with lysis buffer supplemented with $20 \mathrm{mM}$ imidazole, and the elution fractions were collected from lysis buffer containing $250 \mathrm{mM}$ imidazole. The elution fractions were then bufferexchanged with lysis buffer lacking imidazole using Vivaspin® 20 Ultrafiltration Units (Sartorius, Goettingen, Germany) with a molecular weight cutoff of $10 \mathrm{kDa}$. The final buffer imidazole 
concentrations were calculated to be $\sim 0.1 \mathrm{mM}$. Purified and buffer-exchanged protein fractions were then visualized using $10 \%$ SDS-PAGE with Coomassie dye staining.

\section{Patient Sample Collection}

Samples were collected as previously described ${ }^{9}$. Briefly, the UC Irvine Experimental Tissue Resource (ETR) operates under a blanket IRB protocol (UCI \#2012-8716) which enables sample collection in excess of requirements for clinical diagnosis, and allows distribution to investigators. Plasma was collected from daily blood draws of $\operatorname{COVID}(+)$ patients, initially confirmed with pharyngeal swabs. After immediate centrifugation, plasma from heparinanticoagulated blood was stored for $3-4$ days at $4{ }^{\circ} \mathrm{C}$ prior to its release for research use. Personal health information was omitted and unique de-identifier codes were assigned to patients to comply with the Non-Human Subjects Determination exemption from the UCI IRB. At the research facility, SARS-CoV-2 virus in plasma samples was inactivated through treatment by incubation in a $56^{\circ} \mathrm{C}$ water bath for $30 \mathrm{~min}^{24}$ prior to storage at $-80^{\circ} \mathrm{C}$.

\section{Phage ELISAs}

As described in previous reports ${ }^{9}$, pooled plasma from five aEp9(+) patients, five aEp9() patients, or healthy patients (Sigma-Aldrich, Saint Louis, MO) were separately prepared in coating buffer (50 $\mathrm{mM} \mathrm{Na}_{2} \mathrm{CO}_{3}, \mathrm{pH} 9.6$ ); the plasma was diluted 100-fold during this step. Plasma samples were then immobilized in 96 well microtiter plates by shaking the plasma solutions at $150 \mathrm{rpm}$ at room temperature (RT) for $30 \mathrm{~min}$. After aspiration and washing by plate washer (BioTek, Winooski, VT), each well was blocked with $100 \mu \mathrm{L}$ of ChonBlock Blocking Buffer (CBB) (Chondrex, Inc., Woodinville, WA) for $30 \mathrm{mins}$, shaking at $150 \mathrm{rpm}$ at RT. Wells were subsequently washed three times with PBS-T (0.05\% v/v Tween-20 in PBS). Next, 1 nM phagedisplayed candidate "original" epitopes and controls prepared in CBB was incubated in microtiter wells for $1 \mathrm{~h}$ at RT with shaking at $150 \mathrm{rpm}$. Unbound phage were aspirated and removed using 
three washes with PBS-T. The peroxidase-conjugated detection antibody, aM13-HRP (Creative Diagnostics, Shirley, NY), was diluted 1000-fold in Chonblock Secondary Antibody Dilution (Chondrex, Inc., Woodinville, WA) buffer; $100 \mu$ l of this solution was added to each well before incubation for $30 \mathrm{~min}$ at RT with shaking at $150 \mathrm{rpm}$. Following aspiration and three washes (100 $\mu$ each), 1-Step Ultra TMB-ELISA Substrate Solution (ThermoScientific, Carlsbad, CA) was added (100 $\mu$ l per well). Absorbance of TMB substrate was measured twice at $652 \mathrm{~nm}$ by UV-Vis plate reader (BioTek Winooski, VT) after 5 and 15 min of incubation. The experiment was repeated three times using plasma from different $\alpha \mathrm{Ep} 9(+)$ and $\alpha \mathrm{Ep9}(-)$ patients for each experiments, using a total of 15 patients for each group. Each experiment was conducted in technical duplicate.

\section{aEp9 IgG and IgM ELISA}

Plasma from 34 patients, previously tested for the presence of aEp9 Abs using phage ELISAs $^{9}$, were used to test levels of $\alpha$ Ep9 IgGs and IgMs. $2 \mu \mathrm{M}$ eGFP-Ep9 or eGFP-FLAG in PBS pH 8.0 were immobilized onto 96 well microtiter plates via overnight incubation with shaking at $150 \mathrm{rpm}$ at $4^{\circ} \mathrm{C}$. Excess protein was aspirated and removed with three consecutive PBS-T washes. Wells were blocked by adding CBB (100 $\mu \mathrm{l})$ before incubation at $30 \mathrm{~min}$ at RT with shaking at $150 \mathrm{rpm}$. Next, aEp9(+) patient plasma, diluted 1:100 in CBB (100 $\mu \mathrm{l})$, was added to duplicate wells before incubation at RT for $1 \mathrm{~h}$ with shaking at $150 \mathrm{rpm}$. The solutions were discarded and sample wells were washed with PBS-T three times. aEp9 Abs binding to the potential epitopes was detected using horse radish peroxidase (HRP) conjugated aHuman Fc $\lg G$ (Thermo Fisher Scientific, Waltham MA) or algM $\mu$-chain specific (Millipore Sigma, Temecula, CA) Abs diluted 1:5000 in ChonBlock Sample Antibody Dilution buffer. $100 \mu$ l of detection Abs were added to each sample well, and incubated for $30 \mathrm{~min}$ at RT with shaking at $150 \mathrm{rpm}$. Sample wells were aspirated and washed three times in PBS-T, and the binding signal was detected after addition of TMB substrate $(100 \mu$ l per well). 


\section{Bivalent Abs binding ELISA}

eGFP-Ep9 or eGFP-FLAG was serially diluted (120 nM, 40 nM, 13 nM and 4 nM) in PBS $\mathrm{pH}$ 8.0, and added to the appropriate wells in 96 well microtiter plates, followed by shaking overnight at $150 \mathrm{rpm}$ at $4{ }^{\circ} \mathrm{C}$. Excess unbound protein was removed, and the plate was washed three times in PBS-T. Wells were then blocked in CBB and incubated for $30 \mathrm{~min}$ at RT. After blocking, pooled plasma (100 $\mu \mathrm{l}$ per well) from either five $\alpha \mathrm{Ep9}(+)$ patients, or five non- $\alpha \mathrm{Ep} 9$, $\alpha \mathrm{NP}(+)$ patients, or healthy individuals was added to the appropriate wells. Plasma from pooled patients was diluted 100-fold in CBB. As a positive control aFLAG Ab was used as a 1:2000 dilution in CBB. Samples were incubated for $1 \mathrm{~h}$ at RT with $150 \mathrm{rpm}$ shaking. The solution was removed by aspiration, and the plate and washed three times with PBS-T. Then $1 \mathrm{nM}$ EpNeu displaying phage or the phage negative control with no epitopes displayed was diluted in CBB. $100 \mu \mathrm{l}$ phage solution was added to microtiter wells and incubated for $30 \mathrm{~min}$ at RT with shaking at $150 \mathrm{rpm}$. After aspirating and washing off unbound phage, binding of phage-displayed EpNeu to plasma aEp9 Abs was visualized using aM13-HRP Ab diluted 1:10,000 in ChonBlock Sample Antibody Dilution buffer. Samples were incubated for $30 \mathrm{~min}$ at RT with $150 \mathrm{rpm}$ shaking, and unbound Abs were removed through washing with PBS-T three times before addition of TMB substrate $(100 \mu \mathrm{l})$. Experiments were conducted in technical triplicates and repeated three times with different $\alpha \mathrm{Ep}(+)$ and $\alpha \mathrm{Ep}(-)$ patient samples.

\section{Dose-dependent ELISA}

Wells of microtiter plates were coated with serially diluted concentration of eGFP-Ep9, EpNeu and EpPred or eGFP-FLAG, and incubated overnight at $4{ }^{\circ} \mathrm{C}$ before blocking as described above. Next, pooled plasma (100 $\mu$ l per well) from either five $\alpha E p 9(+)$ patients, or five $\alpha \mathrm{Ep} 9(-)$ patients, or healthy individuals at 1:100 total plasma dilution in CBB was added to the appropriate wells. Samples were incubated for $1 \mathrm{~h}$ at RT with shaking at $150 \mathrm{rpm}$. After incubation, unbound solution was removed, and the plates were washed three times with PBS-T. aEp9 IgG levels were 
407

408

409

410

411

412

414

415

416

417

418

419

420

421

422

423

424

425

426

427

428

429

430

431

432

detected by adding aFc IgG-HRP diluted 1:5000 in ChonBlock Sample Dilution buffer, followed by incubation for $30 \mathrm{~min}$ at RT with shaking at $150 \mathrm{rpm}$, followed by addition of TMB substrate (100 $\mu \mathrm{l}$ per well). Experiments were conducted in technical triplicates and repeated three times with different $\alpha \mathrm{Ep}(+)$ and $\alpha \mathrm{Ep}(-)$ patient samples.

\section{Linear B-cell Epitope Prediction}

Linear epitopes from the Influenza A/Para/128982-IEC/2014(H3N2) neuraminidase protein were predicted using the partial sequence with Accession AIX95025.1 from the National Center for Biotechnology Information's GenBank and the linear B-cell epitope prediction tool, Bepipred 2.0 $0^{13}$ (http://www.cbs.dtu.dk/services/BepiPred-2.0/). The prediction thresholds were set to 0.5 . The specificity and sensitivity of epitope prediction at this threshold is 0.572 and 0.586 , respectively.

\section{Structure-based B-cell epitope prediction}

The structure of Influenza A/Para/128982-IEC/2014(H3N2) neuraminidase protein was modelled using Swiss-Model ${ }^{21}$ (https://swissmodel.expasy.org/interactive). Using the ProMod3 3.2.0 tool $^{15}$, a structural model was generated based on the crystal structure $(2.35 \AA$, PDB 4GZS 1.A) of a homologous H3N2 neuraminidase with $96.39 \%$ sequence identity. Modelling methods and quality assessments are further detailed in the report below.

The structural model of Influenza A/Para/128982-IEC/2014(H3N2) neuraminidase was used to predict structure-based epitopes. Using the in silico online platform Discotope $2.0^{16}$ (http://www.cbs.dtu.dk/services/DiscoTope-2.0/), structure-based epitope propensity scores were calculated to predict likely B-cell epitope residues. The score of -3.7 was set as the threshold for epitope prediction, which estimates a specificity and sensitivity of 0.75 and 0.47 , respectively (Figure S4) 
452 value $<0.05$.

\section{Statistical Analysis}

The ELISA data were analyzed in GraphPad Prism 9 (https://www.graphpad.com). Since the ELISA assays of 21 potential AIM epitopes were conducted over several microtiter plates for repeated experiments, the raw absorbance values for every patient sample were normalized and represented as the ratio of phage negative control to the signal. For heatmaps, two-way Analysis of variance (ANOVA) with a Tukey adjustment for multiple comparisons tests were conducted for the entire dataset of epitopes. For column comparisons of two groups, for example IgM levels and $\lg \mathrm{G}$ levels in the $\mathrm{aEp}(+)$ patients, unpaired, two-tailed, parametric t-tests were applied. Additionally, for column comparisons between more than two groups, for example $\lg \mathrm{M}$ or $\lg \mathrm{G}$ levels groups by weeks PSO, One-way ANOVA with a Tukey adjustment for multiple comparisons tests were used. Where indicated, an ANOVA with a Dunnett's adjustment were performed to compare results to healthy Abs interactions to aEp9(+) patient results. Graphs represent SD error bars for technical replicates, defined as replicates of the same conditions in multiple wells of the same plate. Whereas error bars are shown as SEM when an experiment is repeated with different patient sample sets. Correlations between Ep9 and EpNeu levels in patients were determined by plotting normalized values on an $\mathrm{XY}$ graph and performing a linear Pearson's correlation coefficient test, where a $r$ coefficient between 1.0-0.7 were considered strong correlations, values between 0.7 and 0.5 were considered a moderate correlation, and values below 0.5 were considered a weak correlation ${ }^{25}$. The significance of the correlation was evaluated based on $p$ - 


\section{BIOZENTRUM}

SiB University of Basel

\section{SWISS-MODEL Homology Modelling Report}

\section{Model Building Report}

This document lists the results for the homology modelling project "Untitled Project" submitted to SWISS-MODEL workspace on March 30, 2021, 10:35 a.m.. The submitted primary amino acid sequence is given in Table T1.

If you use any results in your research, please cite the relevant publications:

- Waterhouse, A., Bertoni, M., Bienert, S., Studer, G., Tauriello, G., Gumienny, R., Heer, F.T., de Beer, T.A.P., Rempfer, C., Bordoli, L., Lepore, R., Schwede, T. SWISS-MODEL: homology modelling of protein structures and complexes. Nucleic Acids Res. 46(W1), W296-W303 (2018). IM doi>

- Bienert, S., Waterhouse, A., de Beer, T.A.P., Tauriello, G., Studer, G., Bordoli, L., Schwede, T. The SWISS-MODEL Repository - new features and functionality. Nucleic Acids Res. 45, D313-D319 (2017). (10) (doi)

- Studer, G., Tauriello, G., Bienert, S., Biasini, M., Johner, N., Schwede, T. ProMod3 - A versatile homology modelling toolbox. PLOS Comp. Biol. 17(1), e1008667 (2021). (M) doi>

- Studer, G., Rempfer, C., Waterhouse, A.M., Gumienny, G., Haas, J., Schwede, T. QMEANDisCo - distance constraints applied on model quality estimation. Bioinformatics 36, 1765-1771 (2020). LM dois

- Bertoni, M., Kiefer, F., Biasini, M., Bordoli, L., Schwede, T. Modeling protein quaternary structure of homo- and heterooligomers beyond binary interactions by homology. Scientific Reports 7 (2017). La dois

\section{Results}

The SWISS-MODEL template library (SMTL version 2021-03-25, PDB release 2021-03-19) was searched with BLAST (Camacho et al.) and $\mathrm{HHblits}$ (Steinegger et al.) for evolutionary related structures matching the target sequence in Table T1. For details on the template search, see Materials and Methods. Overall 189 templates were found (Table T2).

\section{Models}

The following model was built (see Materials and Methods "Model Building"):

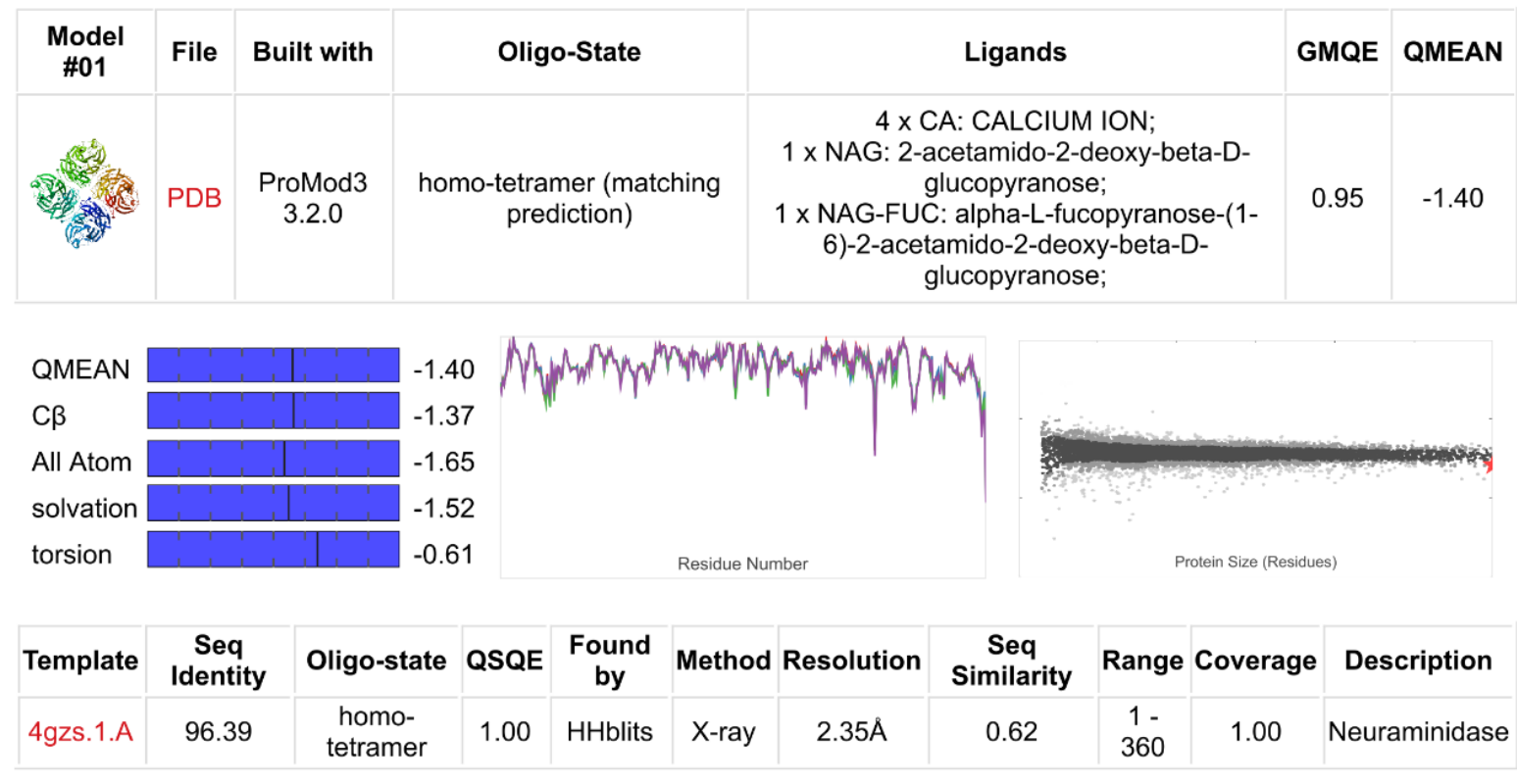

Included Ligands

\begin{tabular}{|c|c|}
\hline Ligand & Description \\
\hline $4 \times$ CA & CALCIUM ION \\
\hline $1 \times$ NAG & 2-acetamido-2-deoxy-beta-D-glucopyranose \\
\hline $1 \times$ NAG-FUC & alpha-L-fucopyranose-(1-6)-2-acetamido-2-deoxy-beta-D-glucopyranose \\
\hline
\end{tabular}


bioRxiv preprint doi: https://doi.org/10.1101/2021.05.21.445201; this version posted June 9, 2021. The copyright holder for this preprint (which was not certified by peer review) is the author/funder. All rights reserved. No reuse allowed without permission.

\begin{tabular}{|c|c|c|}
\hline & & \\
\hline $\begin{array}{l}\text { Ligand } \\
\text { Name.Number }\end{array}$ & $\begin{array}{l}\text { Reason for } \\
\text { Exclusion }\end{array}$ & Description \\
\hline CA.16 & $\begin{array}{l}\text { Not in contact with } \\
\text { model. }\end{array}$ & CALCIUM ION \\
\hline EPE.10 & $\begin{array}{l}\text { Not biologically } \\
\text { relevant. }\end{array}$ & 4-(2-HYDROXYETHYL)-1-PIPERAZINE ETHANESULFONIC ACID \\
\hline EPE.13 & $\begin{array}{l}\text { Not biologically } \\
\text { relevant. }\end{array}$ & 4-(2-HYDROXYETHYL)-1-PIPERAZINE ETHANESULFONIC ACID \\
\hline EPE.18 & $\begin{array}{l}\text { Not biologically } \\
\text { relevant. }\end{array}$ & 4-(2-HYDROXYETHYL)-1-PIPERAZINE ETHANESULFONIC ACID \\
\hline EPE.22 & $\begin{array}{l}\text { Not biologically } \\
\text { relevant. }\end{array}$ & 4-(2-HYDROXYETHYL)-1-PIPERAZINE ETHANESULFONIC ACID \\
\hline NAG.11 & $\begin{array}{l}\text { Clashing with } \\
\text { protein. }\end{array}$ & 2-acetamido-2-deoxy-beta-D-glucopyranose \\
\hline NAG.14 & $\begin{array}{l}\text { Clashing with } \\
\text { protein. }\end{array}$ & 2-acetamido-2-deoxy-beta-D-glucopyranose \\
\hline NAG.15 & $\begin{array}{l}\text { Clashing with } \\
\text { protein. }\end{array}$ & 2-acetamido-2-deoxy-beta-D-glucopyranose \\
\hline NAG.19 & $\begin{array}{l}\text { Clashing with } \\
\text { protein. }\end{array}$ & 2-acetamido-2-deoxy-beta-D-glucopyranose \\
\hline NAG.20 & $\begin{array}{l}\text { Not in contact with } \\
\text { model. }\end{array}$ & 2-acetamido-2-deoxy-beta-D-glucopyranose \\
\hline NAG.24 & $\begin{array}{l}\text { Clashing with } \\
\text { protein. }\end{array}$ & 2-acetamido-2-deoxy-beta-D-glucopyranose \\
\hline NAG-FUC.2 & $\begin{array}{l}\text { Clashing with } \\
\text { protein. }\end{array}$ & alpha-L-fucopyranose-(1-6)-2-acetamido-2-deoxy-beta-D-glucopyranose \\
\hline NAG-FUC.4 & $\begin{array}{l}\text { Clashing with } \\
\text { protein. }\end{array}$ & alpha-L-fucopyranose-(1-6)-2-acetamido-2-deoxy-beta-D-glucopyranose \\
\hline NAG-NAG.8 & $\begin{array}{l}\text { Not in contact with } \\
\text { model. }\end{array}$ & $\begin{array}{l}\text { 2-acetamido-2-deoxy-beta-D-glucopyranose-(1-4)-2-acetamido-2-deoxy-beta-D- } \\
\text { glucopyranose }\end{array}$ \\
\hline $\begin{array}{l}\text { NAG-NAG-BMA- } \\
\text { MAN-MAN.1 }\end{array}$ & $\begin{array}{l}\text { Clashing with } \\
\text { protein. }\end{array}$ & $\begin{array}{c}\text { alpha-D-mannopyranose-(1-3)-[alpha-D-mannopyranose-(1-6)]beta-D- } \\
\text { mannopyranose-(1-4)-2-acetamido-2-deoxy-beta-D-glucopyranose-(1-4)-2- } \\
\text { acetamido-2-deoxy-beta-D-glucopyranose }\end{array}$ \\
\hline $\begin{array}{l}\text { NAG-NAG-BMA- } \\
\text { MAN-MAN.3 }\end{array}$ & $\begin{array}{l}\text { Clashing with } \\
\text { protein. }\end{array}$ & $\begin{array}{c}\text { alpha-D-mannopyranose-(1-3)-[alpha-D-mannopyranose-(1-6)]beta-D- } \\
\text { mannopyranose-(1-4)-2-acetamido-2-deoxy-beta-D-glucopyranose-(1-4)-2- } \\
\text { acetamido-2-deoxy-beta-D-glucopyranose }\end{array}$ \\
\hline $\begin{array}{l}\text { NAG-NAG-BMA- } \\
\text { MAN-MAN.5 }\end{array}$ & $\begin{array}{l}\text { Clashing with } \\
\text { protein. }\end{array}$ & $\begin{array}{c}\text { alpha-D-mannopyranose-(1-3)-[alpha-D-mannopyranose-(1-6)]beta-D- } \\
\text { mannopyranose-(1-4)-2-acetamido-2-deoxy-beta-D-glucopyranose-(1-4)-2- } \\
\text { acetamido-2-deoxy-beta-D-glucopyranose }\end{array}$ \\
\hline $\begin{array}{l}\text { NAG-NAG-BMA- } \\
\text { MAN-MAN.7 }\end{array}$ & $\begin{array}{l}\text { Not biologically } \\
\text { relevant. }\end{array}$ & $\begin{array}{c}\text { alpha-D-mannopyranose-(1-3)-[alpha-D-mannopyranose-(1-6)]beta-D- } \\
\text { mannopyranose-(1-4)-2-acetamido-2-deoxy-beta-D-glucopyranose-(1-4)-2- } \\
\text { acetamido-2-deoxy-beta-D-glucopyranose }\end{array}$ \\
\hline
\end{tabular}

Target AGGDIWVTREPYVSCDPDKGNQFALGQGTTLSKGHSNNTVRDRTPYRTLLMNELGVPFHLGTKQVCIAWSSSSCHDGKAW 4gZs.1.A AGGDIWVTREPYVSCDPDKCYQFALGQGTTLNNVHSNNTVRGRTPYRTLLMNELGVPFHLGTKQVCIAWSSSSCHDGKAW

Target LHVCITGDDKNATASFIYNGRLVDSVVSWSEVLRTQESECVCINGTCTVVMTDGSASGKADTKILFIEEGKIVHTSTLS 4gzs.1.A LHVCITGDDKNATASFIYNGRLVDSWVSWSKEILRTQESECVCINGTCTVVMTDGSASGKADTKILFIEEGKIVHTSTLS

Target GSAQHVEECSCYPRYPGVRCVCRDNWKGSNRPIVDINIKDHSIVSSYVCSGLVGDTPRKNDSSSSGHCLNPNNEEGGHGV 4gZS.1.A GSAQHVEECSCYPRYPGVRCVCRDNWKGSNRPIVDINIKDHSIVSSYVCSGLVGDTPRKNDSSSSSHCLDPNNEEGGHGV

Target KGWAFDDGNDVWMGRTINETSRLGYETFKVIEGWSNPKSKLQINRQVIVDRGDRSGYSGIFSVEGKSCINRCFYVELIRG 4gZs.1.A KGWAFDDGNDVWMGRTINETSRLGYETFKVIEGWSNPKSKLQINRQVIVDRGNRSGYSGIFSVEGKSCINRCFYVELIRG

Target RKEETEVLWTSNSILLFCGTSGTYGTGSWPDAADLNLMPI

4gZS.1.A RKEETEVLWTSNSIVVFCGTSGTYGTGSWPDGADLNLMPI 
bioRxiv preprint doi: https://doi.org/10.1101/2021.05.21.445201; this version posted June 9, 2021. The copyright holder for this preprint (which was not certified by peer review) is the author/funder. All rights reserved. No reuse allowed without permission.

\begin{abstract}
Target AGGDIWVTREPYVSCDPDKGNQFALGQGTTLSKGHSNNTVRDRTPYRTLLMNELGVPFHLGTKQVCIAWSSSSCHDGKAW 4gZS.1.B AGGDIWVTREPYVSCDPDKCYQFALGQGTTLNNVHSNNTVRGRTPYRTLLMNELGVPFHLGTKQVCIAWSSSSCHDGKAW

Target LHVCITGDDKNATASFIYNGRLVDSVVSWSKEVLRTQESECVCINGTCTVVMTDGSASGKADTKILFIEEGKIVHTSTLS 4gZS.1.B LHVCITGDDKNATASFIYNGRLVDSVVSWSKEILRTQESECVCINGTCTVVMTDGSASGKADTKILFIEEGKIVHTSTLS

Target GSAQHVEECSCYPRYPGVRCVCRDNWKGSNRPIVDINIKDHSIVSSYVCSGLVGDTPRKNDSSSSGHCLNPNNEEGGHGV 4gZs.1.B GSAQHVEECSCYPRYPGVRCVCRDNWKGSNRPIVDINIKDHSIVSSYVCSGLVGDTPRKNDSSSSSHCLDPNNEEGGHGV

Target KGWAFDDGNDVWMGRTINETSRLGYETFKVIEGWSNPKSKLQINRQVIVDRGDRSGYSGIFSVEGKSCINRCFYVELIRG 4gZS.1.B KGWAFDDGNDVWMGRTINETSRLGYETFKVIEGWSNPKSKLQINRQVIVDRGNRSGYSGIFSVEGKSCINRCFYVELIRG

Target RKEETEVLWTSNSILLFCGTSGTYGTGSWPDAADLNLMPI

4gzS.1.B RKEETEVLWTSNSIVVFCGTSGTYGTGSWPDGADLNLMPI

Target AGGDIWVTREPYVSCDPDKGNQFALGQGTTLSKGHSNNTVRDRTPYRTLLMNELGVPFHLGTKQVCIAWSSSSCHDGKAW 4gzs.1.C AGGDIWVTREPYVSCDPDKCYQFALGQGTTLNNVHSNNTVRGRTPYRTLLMNELGVPFHLGTKQVCIAWSSSSCHDGKAW

Target LHVCITGDDKNATASFIYNGRLVDSVVSWSKEVLRTQESECVCINGTCTVVMTDGSASGKADTKILFIEEGKIVHTSTLS 4gzs.1.C LHVCITGDDKNATASFIYNGRLVDSVVSWSKEILRTQESECVCINGTCTVVMTDGSASGKADTKILFIEEGKIVHTSTLS

Target GSAQHVEECSCYPRYPGVRCVCRDNWKGSNRPIVDINIKDHSIVSSYVCSGLVGDTPRKNDSSSSGHCLNPNNEEGGHGV 4gZS.1.C GSAQHVEECSCYPRYPGVRCVCRDNWKGSNRPIVDINIKDHSIVSSYVCSGLVGDTPRKNDSSSSSHCLDPNNEEGGHGV

Target KGWAFDDGNDVWMGRTINETSRLGYETFKVIEGWSNPKSKLQINRQVIVDRGDRSGYSGIFSVEGKSCINRCFYVELIRG 4gZs.1.C KGWAFDDGNDVWMGRTINETSRLGYETFKVIEGWSNPKSKLQINRQVIVDRGNRSGYSGIFSVEGKSCINRCFYVELIRG

Target RKEETEVLWTSNSILLFCGTSGTYGTGSWPDAADLNLMPI

4gzs.1.C RKEETEVLWTSNSIVVFCGTSGTYGTGSWPDGADLNLMPI

Target AGGDIWVTREPYVSCDPDKGNQFALGQGTTLSKGHSNNTVRDRTPYRTLLMNELGVPFHLGTKQVCIAWSSSSCHDGKAW 4gZS.1.D AGGDIWVTREPYVSCDPDKCYQFALGQGTTLNNVHSNNTVRGRTPYRTLLMNELGVPFHLGTKQVCIAWSSSSCHDGKAW

Target LHVCITGDDKNATASFIYNGRLVDSVVSWSKEVLRTQESECVCINGTCTVVMTDGSASGKADTKILFIEEGKIVHTSTLS 4gzs.1.D LHVCITGDDKNATASFIYNGRLVDSVVSWSKEILRTQESECVCINGTCTVVMTDGSASGKADTKILFIEEGKIVHTSTLS

Target GSAQHVEECSCYPRYPGVRCVCRDNWKGSNRPIVDINIKDHSIVSSYVCSGLVGDTPRKNDSSSSGHCLNPNNEEGGHGV 4gZS.1.D GSAQHVEECSCYPRYPGVRCVCRDNWKGSNRPIVDINIKDHSIVSSYVCSGLVGDTPRKNDSSSSSHCLDPNNEEGGHGV

Target KGWAFDDGNDVWMGRTINETSRLGYETFKVIEGWSNPKSKLQINRQVIVDRGDRSGYSGIFSVEGKSCINRCFYVELIRG 4gZS.1.D KGWAFDDGNDVWMGRTINETSRLGYETFKVIEGWSNPKSKLQINRQVIVDRGNRSGYSGIFSVEGKSCINRCFYVELIRG

Target RKEETEVLWTSNSILLFCGTSGTYGTGSWPDAADLNLMPI

4gZS.1.D RKEETEVLWTSNSIVVFCGTSGTYGTGSWPDGADLNLMPI
\end{abstract}

\title{
Materials and Methods
}

\section{Template Search}

Template search with BLAST and HHblits has been performed against the SWISS-MODEL template library (SMTL, last update: 2021-03-25, last included PDB release: 2021-03-19).

The target sequence was searched with BLAST against the primary amino acid sequence contained in the SMTL. A total of 103 templates were found. 
bioRxiv preprint doi: https://doi.org/10.1101/2021.05.21.445201; this version posted June 9, 2021. The copyright holder for this preprint (which was not certified by peer review) is the author/funder. All rights reserved. No reuse allowed without permission.

An initial $\mathrm{HHblits}$ profile has been built using the procedure outlined in (Steinegger et al.), followed by 1 iteration of $\mathrm{HHblits}$ against Uniclust30 (Mirdita, von den Driesch et al.). The obtained profile has then be searched against all profiles of the SMTL. A total of 104 templates were found.

\section{Template Selection}

For each identified template, the template's quality has been predicted from features of the target-template alignment. The templates with the highest quality have then been selected for model building.

\section{Model Building}

Models are built based on the target-template alignment using ProMod3 (Studer et al.). Coordinates which are conserved between the target and the template are copied from the template to the model. Insertions and deletions are remodelled using a fragment library. Side chains are then rebuilt. Finally, the geometry of the resulting model is regularized by using a force field.

\section{Model Quality Estimation}

The global and per-residue model quality has been assessed using the QMEAN scoring function (Studer et al.).

\section{Ligand Modelling}

Ligands present in the template structure are transferred by homology to the model when the following criteria are met: (a) The ligands are annotated as biologically relevant in the template library, (b) the ligand is in contact with the model, (c) the ligand is not clashing with the protein, (d) the residues in contact with the ligand are conserved between the target and the template. If any of these four criteria is not satisfied, a certain ligand will not be included in the model. The model summary includes information on why and which ligand has not been included.

\section{Oligomeric State Conservation}

The quaternary structure annotation of the template is used to model the target sequence in its oligomeric form. The method (Bertoni et al.) is based on a supervised machine learning algorithm, Support Vector Machines (SVM), which combines interface conservation, structural clustering, and other template features to provide a quaternary structure quality estimate (QSQE). The QSQE score is a number between 0 and 1, reflecting the expected accuracy of the interchain contacts for a model built based a given alignment and template. Higher numbers indicate higher reliability. This complements the GMQE score which estimates the accuracy of the tertiary structure of the resulting model.

\section{References}

- BLAST

Camacho, C., Coulouris, G., Avagyan, V., Ma, N., Papadopoulos, J., Bealer, K., Madden, T.L. BLAST+: architecture and applications. BMC Bioinformatics 10, 421-430 (2009). l(M doi>

- HHblits

Steinegger, M., Meier, M., Mirdita, M., Vöhringer, H., Haunsberger, S. J., Söding, J. HH-suite3 for fast remote homology detection and deep protein annotation. BMC Bioinformatics 20, 473 (2019). (1) doi?

- Uniclust30

Mirdita, M., von den Driesch, L., Galiez, C., Martin, M.J., Söding, J., Steinegger, M. Uniclust databases of clustered and deeply annotated protein sequences and alignments. Nucleic Acids Research 45, D170-D176 (2016). (M

Table T1:

Primary amino acid sequence for which templates were searched and models were built.

AGGDIWVTREPYVSCDPDKGNQFALGQGTTLSKGHSNNTVRDRTPYRTLLMNELGVPEHLGTKQVCIAWSSSSCHDGKAWLHVCITGDDKNATASFIYNG RLVDSVVSWSKEVLRTQESECVCINGTCTVVMT DGSASGKADTKI LF I EEGKIVHTSTLSGSAQHVEECSCYPRY PGVRCVCRDNWKGSNRP IVDINIKD HSIVSSYVCSGLVGDTPRKNDSSSSGHCLNPNNEEGGHGVKGWAFDDGNDVWMGRT INETSRLGYETFKVIEGWSNPKSKLQINRQVIVDRGDRSGYSGI FSVEGKSCINRCFYVELIRGRKEETEVLWTSNS I LLFCGTSGTYGTGSWPDAADLNLMPI

Table T2:

\begin{tabular}{|l|l|c|c|c|c|c|c|c|c|}
\hline Template & $\begin{array}{c}\text { Seq } \\
\text { Identity }\end{array}$ & $\begin{array}{c}\text { Oligo- } \\
\text { state }\end{array}$ & QSQE & $\begin{array}{c}\text { Found } \\
\text { by }\end{array}$ & Method Resolution & $\begin{array}{c}\text { Seq } \\
\text { Similarity }\end{array}$ & Coverage & Description \\
\hline 4gzs.1.A & 96.39 & $\begin{array}{c}\text { homo- } \\
\text { tetramer }\end{array}$ & 1.00 & HHblits & X-ray & $2.35 \AA$ & 0.62 & 1.00 & Neuraminidase \\
\hline 4gzo.1.A & 96.67 & $\begin{array}{c}\text { homo- } \\
\text { tetramer }\end{array}$ & 1.00 & HHblits & X-ray & $2.60 \AA$ & 0.62 & 1.00 & neuraminidase \\
\hline
\end{tabular}


bioRxiv preprint doi: https://doi.org/10.1101/2021.05.21.445201; this version posted June $9,2021$. The copyright holder for this preprint (which was not certified by peer review) is the author/funder. All rights reserved. No reuse allowed without permission.

\begin{tabular}{|c|c|c|c|c|c|c|c|c|c|}
\hline Template & $\begin{array}{c}\text { Seq } \\
\text { Identity }\end{array}$ & $\begin{array}{l}\text { Oligo- } \\
\text { state }\end{array}$ & QSQE & $\begin{array}{c}\text { Found } \\
\text { by }\end{array}$ & Method & Resolution & $\begin{array}{c}\text { Seq } \\
\text { Similarity }\end{array}$ & Coverage & Description \\
\hline 3tia.1.A & 82.78 & $\begin{array}{l}\text { homo- } \\
\text { tetramer }\end{array}$ & 1.00 & HHblits & X-ray & $1.80 \AA$ & 0.57 & 1.00 & Neuraminidase \\
\hline $4 \mathrm{k} 1 \mathrm{j} .1 . \mathrm{A}$ & 82.78 & $\begin{array}{l}\text { homo- } \\
\text { tetramer }\end{array}$ & 1.00 & HHblits & X-ray & $2.20 \AA$ & 0.57 & 1.00 & Neuraminidase \\
\hline 4h52.1.A & 82.78 & $\begin{array}{l}\text { homo- } \\
\text { tetramer }\end{array}$ & 1.00 & HHblits & X-ray & $1.80 \AA$ & 0.57 & 1.00 & Neuraminidase \\
\hline 5huk.1.A & 79.72 & $\begin{array}{l}\text { homo- } \\
\text { tetramer }\end{array}$ & 1.00 & HHblits & X-ray & $2.45 \AA$ & 0.57 & 1.00 & Neuraminidase \\
\hline 6n4d.1.A & 79.17 & $\begin{array}{l}\text { homo- } \\
\text { tetramer }\end{array}$ & 1.00 & HHblits & X-ray & $1.80 \AA$ & 0.56 & 1.00 & Neuraminidase \\
\hline 2bat.1.A & 83.06 & $\begin{array}{l}\text { homo- } \\
\text { tetramer }\end{array}$ & 1.00 & HHblits & X-ray & $2.00 \AA$ & 0.57 & 1.00 & NEURAMINIDASE N2 \\
\hline 1ivg.1.A & 82.78 & $\begin{array}{l}\text { homo- } \\
\text { tetramer }\end{array}$ & 1.00 & HHblits & X-ray & $1.90 \AA$ & 0.57 & 1.00 & $\begin{array}{c}\text { INFLUENZA A SUBTYPE N2 } \\
\text { NEURAMINIDASE }\end{array}$ \\
\hline 1ive.1.A & 82.78 & $\begin{array}{l}\text { homo- } \\
\text { tetramer }\end{array}$ & 1.00 & HHblits & X-ray & $2.40 \AA$ & 0.57 & 1.00 & $\begin{array}{c}\text { INFLUENZA A SUBTYPE N2 } \\
\text { NEURAMINIDASE }\end{array}$ \\
\hline 1inh.1.A & 82.78 & $\begin{array}{l}\text { homo- } \\
\text { tetramer }\end{array}$ & 1.00 & HHblits & X-ray & $2.40 \AA$ & 0.57 & 1.00 & $\begin{array}{c}\text { INFLUENZA A SUBTYPE N2 } \\
\text { NEURAMINIDASE }\end{array}$ \\
\hline 1ing.1.A & 82.78 & $\begin{array}{l}\text { homo- } \\
\text { tetramer }\end{array}$ & 1.00 & HHblits & X-ray & $2.40 \AA$ & 0.57 & 1.00 & $\begin{array}{c}\text { INFLUENZA A SUBTYPE N2 } \\
\text { NEURAMINIDASE }\end{array}$ \\
\hline 6br5.1.A & 95.83 & monomer & - & HHblits & X-ray & $2.04 \AA$ & 0.62 & 1.00 & Neuraminidase \\
\hline 2aep.1.A & 90.56 & $\begin{array}{l}\text { homo- } \\
\text { tetramer }\end{array}$ & 1.00 & HHblits & X-ray & $2.10 \AA$ & 0.60 & 1.00 & neuraminidase \\
\hline 6n6b.1.A & 89.17 & $\begin{array}{l}\text { homo- } \\
\text { tetramer }\end{array}$ & 1.00 & HHblits & X-ray & $2.30 \AA$ & 0.60 & 1.00 & Neuraminidase \\
\hline $4 m w x .1 . B$ & 47.62 & $\begin{array}{l}\text { homo- } \\
\text { tetramer }\end{array}$ & 0.86 & HHblits & X-ray & $1.80 \AA$ & 0.44 & 0.99 & Neuraminidase \\
\hline 4mwj.1.A & 47.90 & $\begin{array}{l}\text { homo- } \\
\text { tetramer }\end{array}$ & 0.85 & HHblits & X-ray & $1.80 \AA$ & 0.44 & 0.99 & Neuraminidase \\
\hline $5 n z 4.1 . A$ & 46.05 & $\begin{array}{l}\text { homo- } \\
\text { tetramer }\end{array}$ & 0.88 & HHblits & X-ray & $1.36 \AA$ & 0.43 & 0.98 & neuraminidase \\
\hline 2b8h.1.A & 47.62 & $\begin{array}{l}\text { homo- } \\
\text { tetramer }\end{array}$ & 0.85 & HHblits & X-ray & $2.20 \AA$ & 0.44 & 0.99 & Neuraminidase \\
\hline $4 m 3 m .1 . A$ & 44.94 & $\begin{array}{l}\text { homo- } \\
\text { tetramer }\end{array}$ & 0.87 & HHblits & X-ray & $2.10 \AA$ & 0.43 & 0.99 & Neuraminidase \\
\hline 5I14.1.A & 47.90 & $\begin{array}{l}\text { homo- } \\
\text { tetramer }\end{array}$ & 0.84 & HHblits & X-ray & $1.90 \AA$ & 0.44 & 0.99 & Neuraminidase \\
\hline $4 \mathrm{mwj} .1 . \mathrm{A}$ & 49.72 & $\begin{array}{l}\text { homo- } \\
\text { tetramer }\end{array}$ & 0.84 & BLAST & X-ray & $1.80 \AA$ & 0.45 & 0.98 & Neuraminidase \\
\hline 2b8h.1.A & 49.43 & $\begin{array}{l}\text { homo- } \\
\text { tetramer }\end{array}$ & 0.84 & BLAST & X-ray & $2.20 \AA$ & 0.45 & 0.98 & Neuraminidase \\
\hline 1nna.1.D & 49.15 & $\begin{array}{l}\text { homo- } \\
\text { tetramer }\end{array}$ & 0.84 & BLAST & X-ray & $2.50 \AA$ & 0.44 & 0.98 & NEURAMINIDASE \\
\hline 4mju.1.A & 45.51 & $\begin{array}{l}\text { homo- } \\
\text { tetramer }\end{array}$ & 0.84 & HHblits & X-ray & $2.35 \AA$ & 0.43 & 0.99 & Neuraminidase \\
\hline $4 \mathrm{nn} 9.1 . \mathrm{A}$ & 49.15 & $\begin{array}{l}\text { homo- } \\
\text { tetramer }\end{array}$ & 0.83 & BLAST & X-ray & $2.30 \AA$ & 0.44 & 0.98 & NEURAMINIDASE N9 \\
\hline 1mwe.1.C & 49.15 & $\begin{array}{l}\text { homo- } \\
\text { tetramer }\end{array}$ & 0.83 & BLAST & X-ray & $1.70 \AA$ & 0.44 & 0.98 & NEURAMINIDASE \\
\hline 6pzw.1.A & 47.90 & $\begin{array}{l}\text { homo- } \\
\text { tetramer }\end{array}$ & 0.87 & HHblits & EM & NA & 0.44 & 0.99 & Neuraminidase \\
\hline 6pze.1.A & 47.90 & $\begin{array}{l}\text { homo- } \\
\text { tetramer }\end{array}$ & 0.83 & HHblits & X-ray & $2.30 \AA$ & 0.44 & 0.99 & Neuraminidase \\
\hline 6pze.1.A & 49.72 & $\begin{array}{l}\text { homo- } \\
\text { tetramer }\end{array}$ & 0.83 & BLAST & X-ray & $2.30 \AA$ & 0.45 & 0.98 & Neuraminidase \\
\hline
\end{tabular}


bioRxiv preprint doi: https://doi.org/10.1101/2021.05.21.445201; this version posted June 9, 2021. The copyright holder for this preprint (which was not certified by peer review) is the author/funder. All rights reserved. No reuse allowed without permission.

\begin{tabular}{|c|c|c|c|c|c|c|c|c|c|}
\hline Template & $\begin{array}{c}\text { Seq } \\
\text { Identity }\end{array}$ & $\begin{array}{l}\text { Oligo- } \\
\text { state }\end{array}$ & QSQE & $\underset{\text { by }}{\text { Found }}$ & Method & Resolution & $\begin{array}{c}\text { Seq } \\
\text { Similarity }\end{array}$ & Coverage & Description \\
\hline 6pzf.1.A & 47.90 & $\begin{array}{l}\text { homo- } \\
\text { tetramer }\end{array}$ & 0.84 & HHblits & X-ray & $2.80 \AA$ & 0.44 & 0.99 & Neuraminidase \\
\hline 1nca.2.C & 49.15 & $\begin{array}{l}\text { homo- } \\
\text { tetramer }\end{array}$ & 0.84 & BLAST & X-ray & $2.50 \AA$ & 0.44 & 0.98 & $\begin{array}{c}\text { INFLUENZA A SUBTYPE N9 } \\
\text { NEURAMINIDASE }\end{array}$ \\
\hline 1ncd.1.A & 47.90 & $\begin{array}{l}\text { homo- } \\
\text { tetramer }\end{array}$ & 0.84 & HHblits & X-ray & $2.90 \AA$ & 0.44 & 0.99 & $\begin{array}{c}\text { INFLUENZA A SUBTYPE N9 } \\
\text { NEURAMINIDASE }\end{array}$ \\
\hline 1nmb.1.A & 47.90 & $\begin{array}{l}\text { homo- } \\
\text { tetramer }\end{array}$ & 0.81 & HHblits & X-ray & $2.20 \AA$ & 0.44 & 0.99 & N9 NEURAMINIDASE \\
\hline 4d8s.1.A & 45.79 & $\begin{array}{l}\text { homo- } \\
\text { tetramer }\end{array}$ & 0.88 & HHblits & X-ray & $2.40 \AA$ & 0.43 & 0.99 & Neuraminidase \\
\hline 4gb1.1.A & 45.79 & $\begin{array}{l}\text { homo- } \\
\text { tetramer }\end{array}$ & 0.85 & HHblits & X-ray & $2.62 \AA$ & 0.43 & 0.99 & Neuraminidase \\
\hline $4 b 7 q .1 . A$ & 46.18 & $\begin{array}{l}\text { homo- } \\
\text { tetramer }\end{array}$ & 0.85 & HHblits & X-ray & $2.73 \AA$ & 0.43 & 0.98 & NEURAMINIDASE \\
\hline 3o9j.1.A & 45.92 & $\begin{array}{l}\text { homo- } \\
\text { tetramer }\end{array}$ & 0.84 & HHblits & X-ray & $2.00 \AA$ & 0.43 & 0.99 & Neuraminidase \\
\hline 4mjv.1.A & 45.79 & $\begin{array}{l}\text { homo- } \\
\text { tetramer }\end{array}$ & 0.83 & HHblits & X-ray & $2.65 \AA$ & 0.43 & 0.99 & Neuraminidase \\
\hline 2ht7.1.A & 45.51 & $\begin{array}{l}\text { homo- } \\
\text { tetramer }\end{array}$ & 0.81 & HHblits & X-ray & $2.60 \AA$ & 0.43 & 0.99 & Neuraminidase \\
\hline $4 \mathrm{ks} 5.1 . \mathrm{A}$ & 44.94 & $\begin{array}{l}\text { homo- } \\
\text { tetramer }\end{array}$ & 0.77 & HHblits & X-ray & $2.70 \AA$ & 0.43 & 0.99 & Neuraminidase \\
\hline 6crd.1.D & 49.15 & $\begin{array}{l}\text { homo- } \\
\text { tetramer }\end{array}$ & 0.75 & BLAST & X-ray & $2.57 \AA$ & 0.44 & 0.98 & Tetrabrachion,Neuraminidase \\
\hline 6crd.1.B & 49.15 & $\begin{array}{l}\text { homo- } \\
\text { tetramer }\end{array}$ & 0.75 & BLAST & X-ray & $2.57 \AA$ & 0.44 & 0.98 & Tetrabrachion,Neuraminidase \\
\hline 6crd.1.C & 49.15 & $\begin{array}{l}\text { homo- } \\
\text { tetramer }\end{array}$ & 0.75 & BLAST & X-ray & $2.57 \AA$ & 0.44 & 0.98 & Tetrabrachion,Neuraminidase \\
\hline 1ncc.1.A & 49.15 & $\begin{array}{l}\text { homo- } \\
\text { tetramer }\end{array}$ & 0.86 & BLAST & X-ray & $2.50 \AA$ & 0.44 & 0.98 & $\begin{array}{c}\text { INFLUENZA A SUBTYPE N9 } \\
\text { NEURAMINIDASE }\end{array}$ \\
\hline 1ncd.1.A & 49.43 & $\begin{array}{l}\text { homo- } \\
\text { tetramer }\end{array}$ & 0.83 & BLAST & X-ray & $2.90 \AA$ & 0.45 & 0.98 & $\begin{array}{c}\text { INFLUENZA A SUBTYPE N9 } \\
\text { NEURAMINIDASE }\end{array}$ \\
\hline 1nmb.1.A & 49.43 & $\begin{array}{l}\text { homo- } \\
\text { tetramer }\end{array}$ & 0.81 & BLAST & X-ray & $2.20 \AA$ & 0.45 & 0.98 & N9 NEURAMINIDASE \\
\hline 6lxk.1.A & 48.13 & $\begin{array}{l}\text { homo- } \\
\text { tetramer }\end{array}$ & 0.84 & BLAST & X-ray & $3.61 \AA$ & 0.44 & 0.96 & Neuraminidase \\
\hline 1nma.1.A & 47.62 & $\begin{array}{l}\text { homo- } \\
\text { tetramer }\end{array}$ & 0.68 & HHblits & X-ray & $3.00 \AA$ & 0.44 & 0.99 & N9 NEURAMINIDASE \\
\hline 1nma.1.A & 49.43 & $\begin{array}{l}\text { homo- } \\
\text { tetramer }\end{array}$ & 0.65 & BLAST & X-ray & $3.00 \AA$ & 0.45 & 0.98 & N9 NEURAMINIDASE \\
\hline
\end{tabular}

The table above shows the top 50 filtered templates. A further 83 templates were found which were considered to be less suitable for modelling than the filtered list.

1a4g.1.A, 1b9v.1.D, 1inf.1.A, 1iny.1.A, 1I7g.1.A, 1mwe.1.C, 1nca.2.C, 1ncb.1.A, 1ncc.1.A, 1nna.1.D, 1vcj.1.A, 2ht7.1.A, 2htv.1.A, 2hu0.1.A, 2qwd.1.A, 3b7e.1.A, 3cl0.1.A, 3cl2.1.A, 3cye.1.A, 3f14.1.A, 3k36.1.A, 3k38.1.A, 3nn9.1.A, 3o9j.1.A, 3sal.1.A, 4b7m.1.C, 4b7q.1.A, 4b7r.1.A, 4cpl.1.A, 4cpo.1.A, 4d8s.1.A, 4fvk.1.A, 4gb1.1.A, 4gdi.1.A, 4gdi.1.B, 4gdj.1.A, 4gez.1.A, 4h53.1.A, 4h53.1.D, 4hzv.1.A, 4hzy.1.A, 4hzz.1.A, 4k3y.1.A, 4k3y.1.C, 4ks5.1.A, 4m3m.1.A. 4mc7.1.A, 4mju.1.A, 4mjv.1.A, 4mwx.1.B, 4nn9.1.A, 4qn3.1.A, 4qn4.1.A, 4qnp.1.A, 4wa5.1.D, 5hug.1.A, 5hum.1.A, 5hun.1.A, 5I14.1.A, 5nn9.1.A, 5nwe.1.A, 5nz4.1.A, 5nze.1.A, 5nzf.1.A, 5nzn.1.A, 6crd.1.A, 6crd.1.B, 6crd.1.C, 6crd.1.D, 6d96.1.A, 6g01.2.D, 6hfy.1.D, 6hp0.1.C, 6lxi.1.A, 6lxk.1.A, 6nn9.1.A, 6pzf.1.A, 6pzw.1.A, 6q20.1.A, 6q23.1.A, 6v4n.1.A, 6v4o.1.A, 7cm1.1.A 
Table S1 | Potential "original” epitopes targeted by aEp9 Abs

\begin{tabular}{|c|c|c|c|c|c|c|}
\hline Construct \# & Pathogen & Target protein & Accession No. & Residues & Epitope sequences & Similarity \% \\
\hline Phage-disple & constructs & & & & & $\begin{array}{c}\text { template: Ep9 } \\
\text { or EpNeu }\end{array}$ \\
\hline 1 & SARS-COV-2 (Ep9) & Nucleocapsid & QQX29443.1 & $152-172$ & ANNAAIVLQLPQGTTLPKGFY & - \\
\hline 2 & SARS-COV-1 & Nucleocapsid & YP_009825061.1 & $153-173$ & NNNAATVLQLPQGTTLPKGFY & 90.5 \\
\hline 3 & MERS & Nucleocapsid & YP_009047211.1 & 141-151 & NNDSAIVTQFAPGTKLPKNFH & 66.7 \\
\hline 4 & Human coronavirus HKU1 & Nucleocapsid & YP_173242.1 & 166-186 & TTQEAIPTRFPPGTILPQGYY & 57.1 \\
\hline 5 & Human coronavirus NL63 & Nucleocapsid & YP_003771.1 & $119-136$ & NQKPLEPKFSIALPPELS & 13.8 \\
\hline 6 & Human coronavirus OC43 & Nucleocapsid & YP_009555245.1 & $167-187$ & SSDEAIPTRFPPGTVLPQGYY & 71.4 \\
\hline 7 & Human coronavirus $229 \mathrm{E}$ & Nucleocapsid & AGW80953.1 & $122-138$ & SEPEIPHFNQKLPNGVT & 21.4 \\
\hline 8 & Human adenovirus 61 & Hexon & AQQ81927.1 & 123-164 & ANNAATPQVVFYTEDVNLEMPDTHLVFKPAVPNGTIASESLL & 17.6 \\
\hline 9 & Human mastadenovirus $\mathrm{E}$ & PVIII & YP_068038.1 & $76-114$ & AALVYQEIPQPTTVLLPRDAQAEVQLTNSGVQLAGGATL & 31 \\
\hline 10 & Influenza A virus (A/Utah/40/2017) & PB2 polymerase & AVH77902.1 & $225-244$ & GSVYIEVLHLTQGTCWEQMY & 41.7 \\
\hline 11 & $\begin{array}{l}\text { Influenza A virus (EpNeu) } \\
(\mathrm{A} / \mathrm{Para} / 128982-\mathrm{IEC} / 2014(\mathrm{H} 3 \mathrm{~N} 2)\end{array}$ & Neuraminidase, partial & AIX95025.1 & $34-46$ & ALGQGTTLSKGHS & 38.1 \\
\hline 12 & $\begin{array}{l}\text { Influenza B virus } \\
\text { (B/California/88/2019) }\end{array}$ & Neuraminidase & QIA55965.1 & $67-79$ & ATKGVVLLLPEPE & 28.6 \\
\hline 13 & $\begin{array}{l}\text { Influenza C virus } \\
\text { (C/Singapore/DSO-070193/2006) }\end{array}$ & Polymerase PB1, partial & AFV68302.1 & $119-145$ & AATALQLTVDAIKETEGPFKGTTMLEY & 34.4 \\
\hline 14 & Human respiratory syncytial virus $A$ & Fusion protein & ASU44644.1 & $87-100$ & NNAVTELQLLMQST & 38.1 \\
\hline 15 & Human respiratory syncytial virus $A$ & Attachment glycoprotein & ART28426.1 & $106-116$ & GTTPQSTTIPA & 28.6 \\
\hline 16 & Human metapneumovirus & Nucleoprotein, partial & ABO15448.1 & $11-33$ & TTTAVTPSSLPQEITLLCGEILY & 34.8 \\
\hline 17 & Human metapneumovirus & $\begin{array}{l}\text { Attachment glycoprotein, } \\
\text { partial }\end{array}$ & AEW90340.1 & $57-72$ & PQQTTDKHTALPKSIY & 30.8 \\
\hline 18 & Human betaherpesvirus $6 \mathrm{~A}$ & Immediate Early protein 2 & AGJ52064.1 & $1396-1422$ & AATPIDFVGAVKTCNKYAKDNPKEIVL & 10 \\
\hline 19 & Verrucomicrobia bacterium & $\begin{array}{c}\text { NADH-quinone } \\
\text { oxidoreductase (NOX) }\end{array}$ & PYJ45937.1 & $76-89$ & AGVVLQLPQGTTL & 57.1 \\
\hline
\end{tabular}




\begin{tabular}{|c|c|c|c|c|c|c|}
\hline 20 & Clostridium butyricum & \begin{tabular}{|c|} 
Bifunctional \\
methylenetetrahydrofolate \\
dehydrogenase/methenylte \\
trahydrofolate \\
cyclohydrolase (MTHFD2) \\
\end{tabular} & MBE6063617.1 & $94-104$ & IILQLPLPKKF & 47.6 \\
\hline 21 & Fusobacterium mortiferum & Type II secretion protein & WP_118233983.1 & $77-99$ & VENGAIVLQYDKEIYLGLTENFF & 48 \\
\hline 22 & Fusobacterium mortiferum & $\begin{array}{l}\text { Autotransporter outer } \\
\text { membrane protein }\end{array}$ & WP_005886362.1 & $449-460$ & NGAIVGDLVQGT & 38.1 \\
\hline 23 & $\begin{array}{c}\text { Influenza A virus } \\
\text { (A/swine/Missouri/A01774733/2016(H1N2) or } \\
\text { A/Para/129501-IEC/2014(H3N2)) }\end{array}$ & Neuraminidase & ANK78229.1 / AIX95013 & $\begin{array}{c}133-145 / \\
24-36\end{array}$ & ALGQGTTLSNGHS & 92.3 \\
\hline 24 & $\begin{array}{c}\text { Influenza A } \\
\text { (A/swine/Minnesota/A01394278/2013(H3N2)) }\end{array}$ & Neuraminidase & AHA57095.1 & $134-146$ & ALGQGTTLNNGHS & 92.3 \\
\hline 25 & $\begin{array}{ll}\text { Influenza A virus } & \begin{array}{l}(\mathrm{A} / \text { California/04/2009 } \\
(\mathrm{H} 1 \mathrm{~N} 1))\end{array}\end{array}$ & Neuraminidase & AJI76397.1 & $75-89$ & TFFLTQGALLNDKHS & 46.7 \\
\hline 26 & $\begin{array}{l}\text { Influenza A virus } \\
\text { (A/California/111/2015(H3N2)) }\end{array}$ & Neuraminidase & ANM97445.1 & $133-145$ & ALGQGTTLNNVHS & 84.6 \\
\hline 27 & $\begin{array}{c}\text { Influenza A virus } \\
\text { (A/mallard/California/1156/2010(H4N6)) }\end{array}$ & Neuraminidase & AEK50939.1 & 133-142 & ALSQGTTLKG & 84.6 \\
\hline 28 & $\begin{array}{l}\text { Influenza A virus } \\
\text { (A/California/33/2011(H3N2)) }\end{array}$ & Neuramidase & AGL06761.1 & 133-145 & ALGQGTTLSNVHS & 84.6 \\
\hline 29 & $\begin{array}{l}\text { Influenza A virus (EpNeu Pred) } \\
\text { (A/Para/128982-IEC/2014(H3N2)) }\end{array}$ & Neuraminidase, partial & AIX95025.1 & $34-56$ & ALGQGTTLSKGHSNNTVRDRTPY & - \\
\hline \multicolumn{7}{|c|}{ eGFP-fusion constructs } \\
\hline 30 & $\begin{array}{l}\text { Influenza A virus (EpNeu) (A/Para/128982- } \\
\text { IEC/2014(H3N2)) }\end{array}$ & Neuraminidase & AIX95025.1 & $34-46$ & ALGQGTTLSKGHS & - \\
\hline 31 & $\begin{array}{l}\text { Influenza A virus (EpNeu Pred) } \\
\text { (A/Para/128982-IEC/2014(H3N2)) }\end{array}$ & Neuraminidase & AIX95025.1 & $34-56$ & ALGQGTTLSKGHSNNTVRDRTPY & - \\
\hline
\end{tabular}


Table S2 | Primers used to subclone potential original epitopes

\begin{tabular}{|c|c|c|}
\hline Construct \# & Primer names: & Oligonucleotide sequence \\
\hline \multicolumn{3}{|c|}{ Phage-displayed constructs } \\
\hline \multirow[b]{2}{*}{1} & primer_1_F & tcaagggactaccttgcccaaggggttctatGGTGGAGGATCCGGGAGC \\
\hline & primer_1_R & ggtaattgtaacacgattgcagcgttattagcTCCACTTCCTTTATCATCGTCATCTTTATAATC \\
\hline \multirow[b]{2}{*}{2} & primer_2_F & acttccacagggaacgacactgccaaagggatttGGTGGAGGATCCGGGAGC \\
\hline & primer_2_R & tgcagtacagtggcagcattgttatttccacttccTTTATCATCGTCATCTTTATAATCAACCAATGC \\
\hline \multirow[b]{2}{*}{3} & primer_3_F & tccgggtacaaagttaccaaagaacttccacGGTGGAGGATCCGGGAGC \\
\hline & primer_3_R & gcaaattgagtaactatcgctgaatcattgttTCCACTTCCTTTATCATCGTCATCTTTATAATC \\
\hline \multirow[b]{2}{*}{4} & primer_4_F & tcccggaactattttaccccaaggatactatGGTGGAGGATCCGGGAGC \\
\hline & primer_4_R & gggaatctagtgggaatcgcctcctgagtagtTCCACTTCCTTTATCATCGTCATCTTTATAATC \\
\hline \multirow[b]{2}{*}{5} & primer_5_F & agtattgccttgccacctgagttatctGGTGGAGGATCCGGGAGC \\
\hline & primer_5_R & aaatttcggttcaagcggctttgattTCCACTTCCTTTATCATCGTCATCTTTATAATC \\
\hline \multirow[b]{2}{*}{6} & primer_6_F & tccgggtactgtttgcctcagggttactacGGTGGAGGATCCGGGAGC \\
\hline & primer_6_R & ggaaaccgcgttgggatggcctcatcagagctTTTATCATCGTCATCTTTATAATCAACCAATGC \\
\hline \multirow[b]{2}{*}{7} & primer_7_F & ccagaaattgccgaacggcgttactGGTGGAGGATCCGGGAGC \\
\hline & primer_7_R & ttaaagtgagggatctctggctcgctTTTATCATCGTCATCTTTATAATCAACCAATGC \\
\hline \multirow[b]{2}{*}{8} & primer_8_F & TTCTAAATTCACATCCTCAGTATAGAACACGACTTGTGGGGTTGCTGCATTGTTGGCTTTATCATCGTCATCTTTATAATCAACCAATGC \\
\hline & primer_8_R & ATGCCAGATACTCATTTGGTCTTCAAACCTGCGGTCCCGAATGGCACGATTGCTAGTGAATCTCTCCTCggtggaggatccgggagc \\
\hline \multirow[b]{2}{*}{9} & primer_9_F & ACGCGGGAGCAGCACTGTAGTCGGCTGAGGGATTTCCTGGTACACCAGGGCGGCTTTATCATCGTCATCTTTATAATCAACCAATGC \\
\hline & primer_9_R & GACGCGCAAGCCGAGGTCCAGTTGACAAACAGCGGTGTACAATTGGCTGGCGGTGCCACTCTGggtggaggatccgggagc \\
\hline \multirow[b]{2}{*}{10} & primer_10_F & acacaaggcacttgctgggagcaaatgtatGGTGGAGGATCCGGGAGC \\
\hline & primer_10_R & gaggtgcagcacttcaatatagactgacccTTTATCATCGTCATCTTTATAATCAACCAATGC \\
\hline \multirow[b]{2}{*}{11} & primer_11_F & gcttctaagggccacagcGGTGGAGGATCCGGGAGC \\
\hline & primer_11_R & gtggtgccttggcccaatgcTTTATCATCGTCATCTTTATAATCAACCAATGC \\
\hline \multirow[b]{2}{*}{12} & primer_12_F & gctgcttccggagccagagGGTGGAGGATCCGGGAGC \\
\hline & primer_12_R & agcacgacgcctttcgtagcTTTATCATCGTCATCTTTATAATCAACCAATGC \\
\hline \multirow[b]{2}{*}{13} & primer_13_F & gactgaaggtccttttaagggcacgacaatgttggaatatGGTGGAGGATCCGGGAGC \\
\hline & primer_13_R & tctttaatggcatcgaccgtaagttggagagccgtcgctgcTTTATCATCGTCATCTTTATAATCAACCAATGC \\
\hline \multirow[b]{2}{*}{14} & primer_14_F & caactcctcatgcagtctacaGGTGGAGGATCCGGGAGC \\
\hline & primer_14_R & aagttctgtgacagcattgttTTTATCATCGTCATCTTTATAATCAACCAATGC \\
\hline & primer_15_F & gacaaccatccctgcaGGTGGAGGATCCGGGAGC \\
\hline 15 & primer_15_R & gattgaggcgtagtcccTTTATCATCGTCATCTTTATAATCAACCAATGC \\
\hline & primer_16_F & ggaaatcactctcttgtgcggcgaaatcttatatGGTGGAGGATCCGGGAGC \\
\hline 16 & primer_16_R & tgcggcaggctagatggcgtaactgccgtagtagtTTTATCATCGTCATCTTTATAATCAACCAATGC \\
\hline & primer_17_F & acggcactgccgaagtccatctacGGTGGAGGATCCGGGAGC \\
\hline 17 & primer_17_R & atgcttgtcagtggtctgttgtggTTTATCATCGTCATCTTTATAATCAACCAATGC \\
\hline & primer_18_F & caacaaatacgctaaggacaatccgaaagagatcgtgcttGGTGGAGGATCCGGGAGC \\
\hline 18 & primer_18_R & caggtcttgacagctccgacgaagtcgatgggcgtagccgcTTTATCATCGTCATCTTTATAATCAACCAATGC \\
\hline & primer_19_F & tccacaaggcaccacccttGGTGGAGGATCCGGGAGC \\
\hline 19 & primer_19_R & agctgcagcacgacgccggcTTTATCATCGTCATCTTTATAATCAACCAATGC \\
\hline & primer_20_F & gttacctaagaaattcGGTGGAGGATCCGGGAGC \\
\hline 20 & primer_20_R & ggaagctgcaaaataatTTTATCATCGTCATCTTTATAATCAACCAATGC \\
\hline & primer_21_F & agagatttacctggggctgacagagaactttttGGTGGAGGATCCGGGAGC \\
\hline 21 & primer_21_R & ttgtcgtactgtaatacaattgcgccgttctccacTTTATCATCGTCATCTTTATAATCAACCAATGC \\
\hline & primer_22_F & gatttagtacagggtaccGGTGGAGGATCCGGGAGC \\
\hline 22 & primer_22_R & gcctacaatggcaccattTTTATCATCGTCATCTTTATAATCAACCAATGC \\
\hline & primer_23_F & CGCTTTCTAATGGCCACAGCG \\
\hline 23 & primer_23_R & TGGTGCCTTGGCCCAATG \\
\hline & primer_24_F & CACCACGCTTaacaatGGCCACAGCG \\
\hline 24 & primer_24_R & CCTTGGCCCAATGCTTTAT \\
\hline & primer_25_F & tcttttgaacgacaagcattcaGGTGGAGGATCCGGGAGC \\
\hline 25 & primer_25_R & gctccctgagtcagaaagaatgtTTTATCATCGTCATCTTTATAATCAACCAATGC \\
\hline & primer_26_F & attaaataacgttcatagtGGTGGAGGATCCGGGAGC \\
\hline 26 & primer_26_R & gtagtaccctggcccaaggcTTTATCATCGTCATCTTTATAATCAACCAATGC \\
\hline & primer_27_F & tgcgaacggtacgattcatgatcgttccccattcGGTGGAGGATCCGGGAGC \\
\hline 27 & primer_27_R & tgacgaccttcagtgtcgtgccttgagataacgcTTTATCATCGTCATCTTTATAATCAACCAATGC \\
\hline & primer_28_F & tttgtcgaatgtacactccGGTGGAGGATCCGGGAGC \\
\hline 28 & primer_28_R & gtggtaccctggccaagagcTTTATCATCGTCATCTTTATAATCAACCAATGC \\
\hline & primer_29_F & ctcgaacaacaccgttcgcgaccgtactccgtacGGTGGAGGATCCGGGAGC \\
\hline 29 & primer_29_R & tgccctttagacagagttgtcccttggcccaaagcTTTATCATCGTCATCTTTATAATCAACCAATGC \\
\hline eGFP-fusion c & nstructs & \\
\hline & primer_30_F & gcttctaagggccacagcAGCGGAAGTGGAGATTATAAAGATGAC \\
\hline 30 & primer_30_R & gtggtgccttggeccaatgcGGAGCTCCCGGATCCTCC \\
\hline & primer_31_F & ctcgaacaataccgtccgcgatcggactccgtacAGCGGAAGTGGAGATTATAAAGATGAC \\
\hline 31 & primer_31_R & tgacccttgctcaaagtagtaccctgcccaagcgcGGAGCTCCCGGATCCTCC \\
\hline
\end{tabular}


471

A

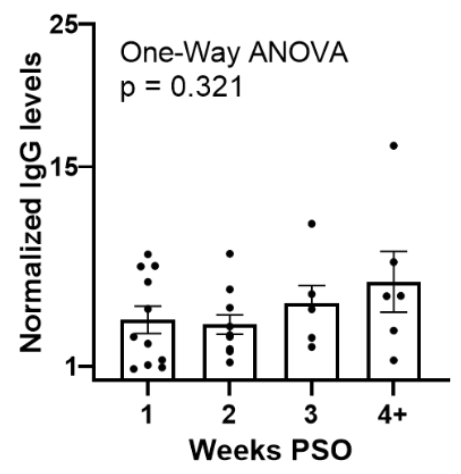

B

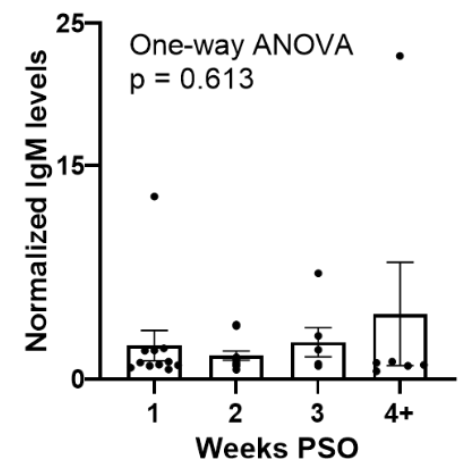

Figure S1 | Early upregulation of aEp9 IgGs. ELISA of aEp9 (A) IgG and (B) IgM levels in aEp9(+) patients $(n=34)$ from plasma collected at the indicated time periods post-symptom onset (PSO). Statistical analysis was conducted using one-way ANOVA, ad hoc Tukey test. Error bars represent SEM.

A

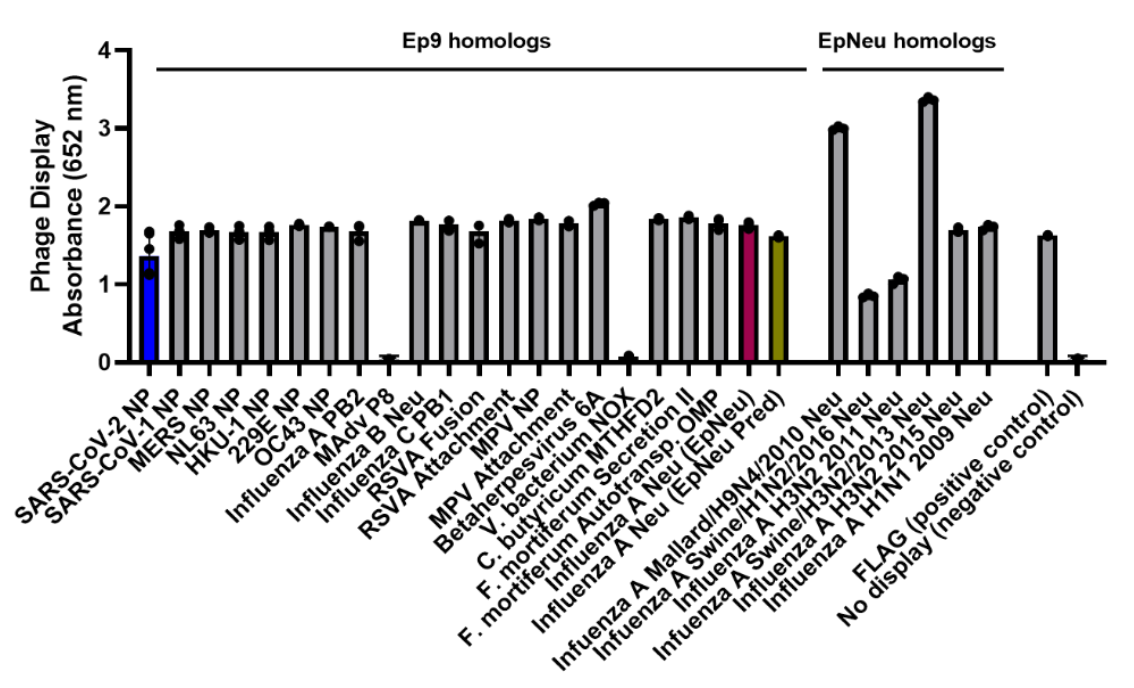

B

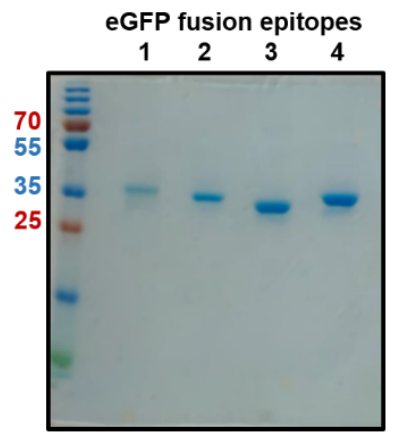

L: PageRuler ${ }^{\mathrm{TM}}$ Plus Prestained ladder 1: EpNeu Pred

2: EpNeu

3: FLAG neg ctr 4: Ep9

Figure S2 | Expression of phage-displayed and eGFP-fused potential AIM epitopes. to the N-terminus of the P8 coat protein. Immobilized aFLAG Abs in microtiter wells bind the displayed FLAG-tag and epitope, and binding is detected with $\alpha \mathrm{M}-13-\mathrm{HRP}$ Abs as usual. Phage with no epitope displayed provide the negative control. Epitopes for mastadenovirus protein (mAdV) P8 and $V$. bacterium NADH oxidoreductase (NOX) did not display. Error bars represent SD values. (B) 10\% SDS-PAGE gel stained with epitopes, EpPred, EpNeu, FLAG negative control and Ep9. 


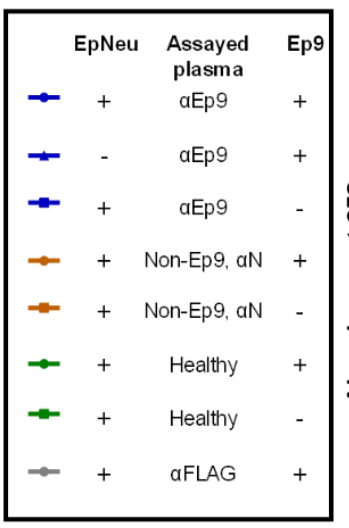

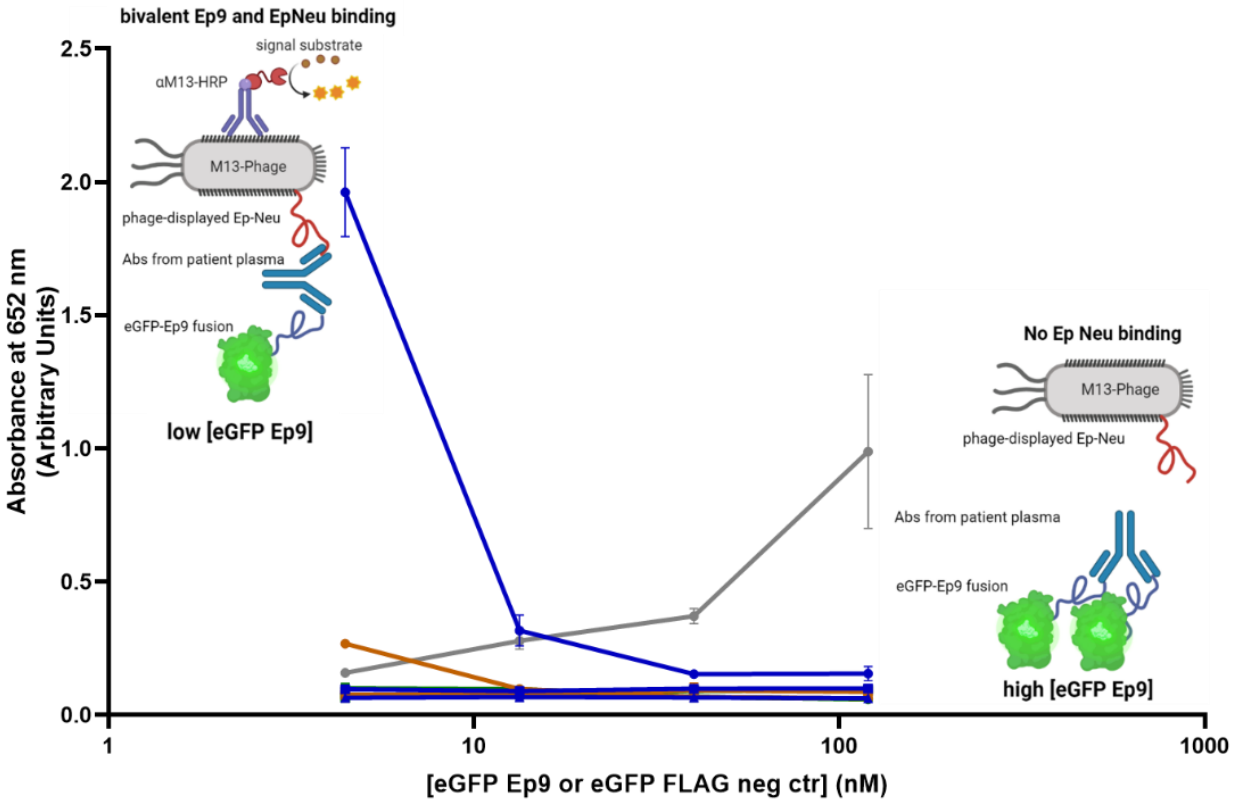

Figure S3 | Optimization of assay to determine cross-reactivity of aEp9 Ab to Ep9 and EpNeu. Sandwich ELISA testing the binding of Abs from the pooled plasma of five aEp9(+) patients, five aEp9(-) patients with other aNP Abs and healthy individuals. This experiment examines bivalent binding to various doses of immobilized eGFP-fused Ep9 epitope (120, 40, 13 and $4 \mathrm{nM}$ ) and phage-displayed EpNeu in solution. The data shows that Abs from aEp9(+) patients, but not aEp9(-) or healthy individuals, bivalently bind both EpNeu and Ep9. The positive control ( $\alpha$ FLAG 1:2000 fold dilution) at $100 \mathrm{nM}$ eGFP demonstrates concentrations appropriate for bivalent binding to immobilized and insolution tags. The schematic diagram illustrates the binding observed for bivalence in aEp9 Abs, where the antibody bridges plate-bound eGFP at its high concentrations. Therefore, Figure 2 in the main text uses 4 nM of eGFP Ep9 coated on the plate, and the FLAG positive control uses eGFP at $100 \mathrm{nM}$. Error bars represent SD. 
A

Linear Epitope Mapping Prediction

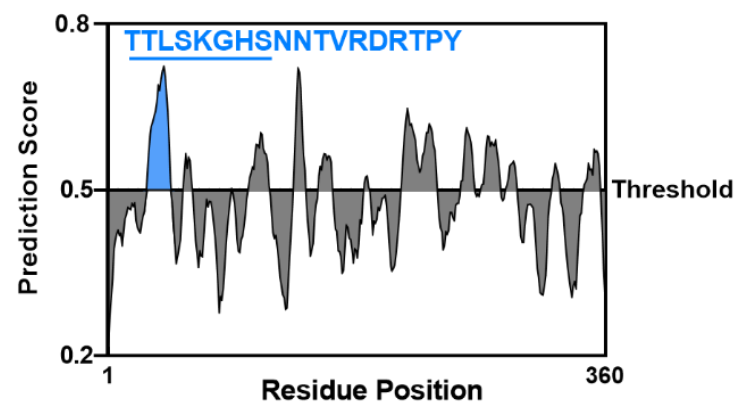

B

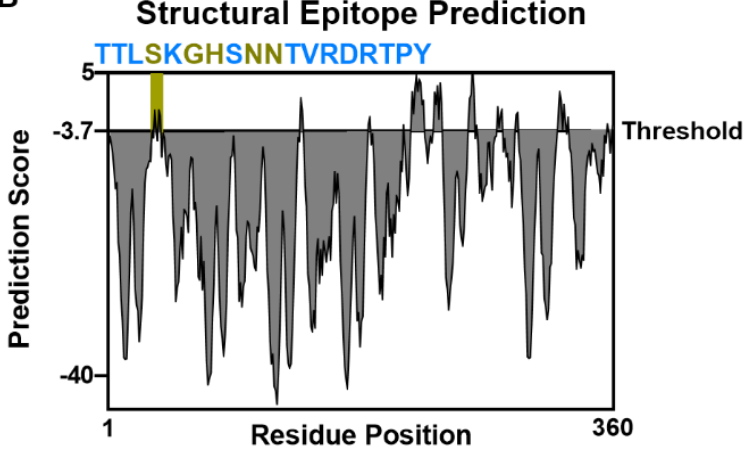

503

504

505

506

507

508

509

510

511

512
Figure S4 | Linear and structural epitope mapping prediction of Influenza A H3N2 Neuraminidase. (A) Linear epitope mapping prediction of the Flu A 2014 H3N2 using Bepipred $2.0^{13}$ demonstrates high prediction scores in a region spanning 18 residues, which includes eight residues from EpNeu (underlined). The additional 10 predicted residues were included as part of an extended epitope termed EpPred. (B) Structural epitope mapping, using Discotope $2.0^{16}$, of the modelled neuraminidase protein from Flu A 2014 H3N2 (SWISS-model ${ }^{21}$ ), predicts an epitope of five residues. These were captured by EpPred, including three found in EpNeu. 


\section{References}

1. Fierz, W. \& Walz, B. Antibody Dependent Enhancement Due to Original Antigenic Sin and the Development of SARS. Front. Immunol. 11, 1120 (2020).

2. Brown, E. L. \& Essigmann, H. T. Original Antigenic Sin: the Downside of Immunological Memory and Implications for COVID-19. mSphere 6, (2021).

3. Kohler, H. \& Nara, P. A Novel Hypothesis for Original Antigenic Sin in the Severe Disease of SARS-CoV-2 Infection. Monoclonal Antibodies in Immunodiagnosis and Immunotherapy 39, 107-111 (2020).

4. $\quad \mathrm{Ng}, \mathrm{K} . \mathrm{W}$. et al. Preexisting and de novo humoral immunity to SARS-CoV-2 in humans. Science (80-. ). 370, 1339-1343 (2020).

5. Anderson, E. M. et al. Seasonal human coronavirus antibodies are boosted upon SARS-CoV-2 infection but not associated with protection. medRxiv 7, 2020.11.06.20227215 (2020).

6. Focosi, D. et al. Previous humoral immunity to the endemic seasonal alphacoronaviruses NL63 and 229E is associated with worse clinical outcome in COVID-19 and suggests original antigenic sin. Life 11, (2021).

7. Shrock, E. et al. Viral epitope profiling of COVID-19 patients reveals crossreactivity and correlates of severity. Science (80-. ). eabd4250 (2020). doi:10.1126/science.abd4250

8. Wang, L. et al. Serological Responses to Human Virome Define Clinical Outcomes of Italian Patients Infected with SARS-CoV-2. medRxiv Prepr. Serv. Heal. Sci. (2020). doi:10.1101/2020.09.04.20187088

9. Sen, S. R. et al. Predicting COVID-19 Severity with a Specific Nucleocapsid Antibody plus Disease Risk Factor Score. mSphere 6, e00203-21 (2021).

10. Altschul, S. F. et al. Gapped BLAST and PSI-BLAST: A new generation of protein database search programs. Nucleic Acids Research 25, 3389-3402 (1997).

11. Madej, T. et al. MMDB and VAST+: Tracking structural similarities between macromolecular complexes. Nucleic Acids Res. 42, (2014).

12. World Health Organization. WHO | Cumulative Number of Reported Probable Cases of Severe Acute Respiratory Syndrome (SARS). WHO 9-11 (2013). Available at: http://www.who.int/csr/sars/country/en/. (Accessed: 16th May 2021)

13. Jespersen, M. C., Peters, B., Nielsen, M. \& Marcatili, P. BepiPred-2.0: Improving sequence-based B-cell epitope prediction using conformational epitopes. Nucleic Acids Res. 45, W24-W29 (2017).

14. Gentles, L. E., Wan, H., Eichelberger, M. C. \& Bloom, J. D. Antibody neutralization of an influenza virus that uses neuraminidase for receptor binding. Viruses 12, 2020.05.08.084954 (2020).

15. Studer, G. et al. ProMod3 - A versatile homology modelling toolbox. PLoS Comput. Biol. 17, (2021).

16. Kringelum, J. V., Lundegaard, C., Lund, O. \& Nielsen, M. Reliable B Cell Epitope Predictions: Impacts of Method Development and Improved Benchmarking. PLoS Comput. Biol. 8, (2012).

17. Lipničanová, S., Chmelová, D., Godány, A., Ondrejovič, M. \& Miertuš, S. Purification of viral neuraminidase from inclusion bodies produced by recombinant Escherichia coli. J. Biotechnol. 316, 27-34 (2020). 
18. Xie, H. et al. H3N2 Mismatch of 2014-15 Northern Hemisphere Influenza Vaccines and Head-to-head Comparison between Human and Ferret Antisera derived Antigenic Maps. Sci. Rep. 5, 1-10 (2015).

19. Flannery, B. et al. Enhanced Genetic Characterization of Influenza A(H3N2) Viruses and Vaccine Effectiveness by Genetic Group, 2014-2015. in Journal of Infectious Diseases 214, 1010-1019 (Oxford University Press, 2016).

20. Letunic, I. \& Bork, P. Interactive Tree of Life (iTOL) v4: Recent updates and new developments. Nucleic Acids Res. 47, W256-W259 (2019).

21. Waterhouse, A. et al. SWISS-MODEL: Homology modelling of protein structures and complexes. Nucleic Acids Res. 46, W296-W303 (2018).

22. Benchling, I. Benchling [Biology Software]. Retrieved from https://benchling.com (2017).

23. Levin, A. M. Exploring the interaction between the protein kinase A catalytic subunit and caveolin-1 scaffolding domain with shotgun scanning, oligomer complementation, NMR, and docking. Protein Sci. 15, 478-486 (2006).

24. Pastorino, B., Touret, F., Gilles, M., de Lamballerie, X. \& Charrel, R. N. Heat inactivation of different types of SARS-CoV-2 samples: What protocols for biosafety, molecular detection and serological diagnostics? Viruses 12, 735-743 (2020).

25. Mukaka, M. M. Statistics corner: A guide to appropriate use of correlation coefficient in medical research. Malawi Med. J. 24, 69-71 (2012). 